\title{
PARABOLIC CHARACTER SHEAVES, II
}

\author{
G. LuszTiG \\ Dedicated to Boris Feigin on the occasion of his 50th birthday
}

\section{INTRODUCTION}

Let $G$ be a connected reductive algebraic group over an algebraically closed field k. Let $Z$ be the algebraic variety consisting of all triples $\left(P, P^{\prime}, U_{P^{\prime}} g U_{P}\right)$ where $P, P^{\prime}$ run through some fixed conjugacy classes of parabolics in $G$ and $g$ is an element of $G$ that conjugates $P$ to a parabolic in a fixed "good" relative position $y$ with $P^{\prime}$ (here $U_{P}, U_{P^{\prime}}$ are the unipotent radicals of $P, P^{\prime}$ ). The varieties $Z$ include more or less as a special case the boundary pieces of the De ConciniProcesi completion $\bar{G}$ of $G$ (assumed to be adjoint). They also include as a special case the varieties studied in the first part of this series [L9] (where $y=1$ that is, $\left.g P g^{-1}=P^{\prime}\right)$. In this special case a theory of "character sheaves" on $Z$ was developed in [L9]. In the present paper we extend the theory of character sheaves to a general $Z$.

We now review the content of this paper in more detail. (The numbering of sections continues that of [L9]; we also follow the notation of [L9] .)

In Section 8 we introduce a partition of $Z$ similar to that in [L9]; as in [L9], it is based on the combinatorics in Section 2. But whereas in [L9] the combinatorics needed is covered by the results in $[\mathrm{B}]$, for the present paper we actually need the slight generalization of $[\mathrm{B}]$ given in Section 2. Now, it is not obvious that the partition of $Z$ defined in Section 8 reduces for $y=1$ to that in Section 3; this needs an argument that is given in Section 9. In Section 10 we consider the example where $G$ is a general linear group. In Section 11 we define the "parabolic character sheaves" on $Z$. As in the case $y=1$ (Section 4), we give two definitions for these; one uses the partition in Section 8 and allows us to enumerate the parabolic character sheaves; the other one imitates the definition of character sheaves in [L3]. (The two definitions are equivalent by 11.15 and 11.18.) The theory of character sheaves in Section 11 generalizes that in Section 4 (this is seen from the second definition). A consequence of the coincidence of the two definitions of parabolic character sheaves on $Z$ is that a statement like 0.1(a) (concerning characteristic functions over a finite field) continues to hold in the generality of this paper. In

Supported in part by the National Science Foundation

Typeset by $\mathcal{A} \mathcal{M}$ S-TEX 
Section 12 we define the notion of character sheaf on the completion $\bar{G}$. We again have two definitions; one is based on the partition in Section 8, and the second one is reminiscent of the definition of character sheaves in [L3]. We expect that these two definitions coincide, but we cannot prove this; if this was true, we would have an analogue of 0.1 (a) for $\bar{G}$ over a finite field. Our results also provide a finite partition of $\bar{G}$ into $G$-stable pieces (a refinement of the usual partition of $\bar{G}$ into $G \times G$-orbits) which allows us to give an explicit description of the set of $G$-conjugacy classes in $\bar{G}$ (see $12.3(\mathrm{a})$ ).

\section{Contents}

8. The variety $Z_{J, y, \delta}$ and its partition.

9. Comparison of two partitions.

10. Example.

11. Parabolic character sheaves on $Z_{J, y, \delta}$.

12. Completion.

\section{The VARIETy $Z_{J, y, \delta}$ AND ITS PARTITION}

8.1. We preserve the setup of 3.1. (Thus, $\hat{G}$ is a possibly disconnected reductive algebraic group over $\mathbf{k}$ with identity component $G$ and $G^{1}$ is a fixed connected component of $\hat{G}$. Also $W, I$ is the Weyl group of $G$ and $\delta: W \stackrel{\sim}{\rightarrow} W$.) Let $P \in \mathcal{P}_{J}, Q \in \mathcal{P}_{K}, u=\operatorname{pos}(P, Q)$. We have

$$
\operatorname{dim}\left(\left(U_{P} \cap U_{Q}\right) \backslash U_{P}\right)=l(u)+\nu_{J}-\nu_{J \cap \operatorname{Ad}(u) K} .
$$

Here $\nu_{J}$ is the number of reflections in $W_{J}$.

8.2. Let $P, P^{\prime}$ be two parabolics of $G$. The following hold.

(a) $P^{P^{\prime}}, P^{\prime P}$ are in good position and $\operatorname{pos}\left(P, P^{\prime}\right)=\operatorname{pos}\left(P^{P^{\prime}}, P^{\prime P}\right)$;

(b) if $B \in \mathcal{B}, B \subset P^{P^{\prime}}$ then for some $B^{\prime} \in \mathcal{B}, B^{\prime} \subset P^{\prime}$ we have $\operatorname{pos}\left(B, B^{\prime}\right)=$ $\operatorname{pos}\left(P, P^{\prime}\right)$.

To prove (b) we may replace $P, P^{\prime}$ by $P^{P^{\prime}}, P^{\prime P}$. It suffices to prove: if $P, P^{\prime}$ are in good position and $B \subset P$, then for some $B^{\prime} \subset P^{\prime}$ we have $\operatorname{pos}\left(B, B^{\prime}\right)=\operatorname{pos}\left(P, P^{\prime}\right)$.

8.3. If $P, Q$ are parabolics in good position, we have a bijection

$\{$ parabolics contained in $P\} \stackrel{\sim}{\longrightarrow}$ pparabolics contained in $Q\}$

given by $P^{\prime} \leftrightarrow Q^{\prime}, Q^{\prime}=Q^{P^{\prime}}, P^{\prime}=P^{Q^{\prime}}$. (Then $P^{\prime}, Q^{\prime}$ are in good position and $\operatorname{pos}\left(P^{\prime}, Q^{\prime}\right)=\operatorname{pos}(P, Q)$.)

Lemma 8.4. Let $P, Q, R$ be parabolics with a common Levi L. Then $\operatorname{pos}(P, Q) \operatorname{pos}(Q, R)=\operatorname{pos}(P, R)$.

Let $\beta$ be a Borel of $L$. Then $B=U_{P} \beta, B^{\prime}=U_{Q} \beta, B^{\prime \prime}=U_{R} \beta$ are Borels of $P, Q, R$ respectively and we have

$$
\operatorname{pos}(P, Q)=\operatorname{pos}\left(B, B^{\prime}\right), \operatorname{pos}(Q, R)=\operatorname{pos}\left(B^{\prime}, B^{\prime \prime}\right), \operatorname{pos}(P, R)=\operatorname{pos}\left(B, B^{\prime \prime}\right) .
$$

It suffices to show that $\operatorname{pos}\left(B, B^{\prime}\right) \operatorname{pos}\left(B^{\prime}, B^{\prime \prime}\right)=\operatorname{pos}\left(B, B^{\prime \prime}\right)$. This holds since $B, B^{\prime}, B^{\prime \prime}$ contain $\beta$ hence have a common maximal torus. 
Lemma 8.5. Let $P, Q, R$ be parabolics with a common Levi L; let $P^{\prime}, Q^{\prime}, R^{\prime}$ be parabolics with a common Levi $L^{\prime}$. Assume that $\operatorname{pos}(P, Q)=\operatorname{pos}\left(P^{\prime}, Q^{\prime}\right)$, $\operatorname{pos}(Q, R)=\operatorname{pos}\left(Q^{\prime}, R^{\prime}\right)$. Assume also that $P, P^{\prime}$ have the same type; $Q, Q^{\prime}$ have the same type; $R, R^{\prime}$ have the same type. Then there exists $x \in G$ that conjugates $P, Q, R$ to $P^{\prime}, Q^{\prime}, R^{\prime}$.

Clearly, we can assume that $P=P^{\prime}, Q=Q^{\prime}, L=L^{\prime}$. Then we use the following fact: if $Q, R, R^{\prime}$ are parabolics with a common Levi $L$ such that $\operatorname{pos}(Q, R)=$ $\operatorname{pos}\left(Q, R^{\prime}\right)$ then $R=R^{\prime}$. (This can be reduced to the case where $Q, R, R^{\prime}$ are Borels, which is clear.)

Lemma 8.6. Let $Q, Q^{\prime}$ be parabolics with a common Levi. Then $Q \cap U_{Q^{\prime}}=$ $U_{Q} \cap U_{Q^{\prime}}$.

It suffices to show that Lie $Q \cap$ Lie $U_{Q^{\prime}}=$ Lie $U_{Q} \cap$ Lie $U_{Q^{\prime}}$. Let $L$ be a common Levi of $Q, Q^{\prime}$. Consider the weight decomposition Lie $G=\oplus_{\alpha}$ Lie $G_{\alpha}$ with respect to the connected centre of $L$. Then Lie $G_{0}=$ Lie $L$ and Lie $U_{Q}$, Lie $U_{Q^{\prime}}$ are direct sums of various Lie $G_{\alpha}$ with $\alpha \neq 0$. Let $x \in$ Lie $Q \cap$ Lie $U_{Q^{\prime}}$. We have $x=x_{0}+x^{\prime}$ with $x_{0} \in$ Lie $L^{\prime}$ and $x^{\prime} \in$ Lie $U_{Q}$ is in $\oplus_{\alpha \neq 0}$ Lie $G_{\alpha}$. Since $x \in$ Lie $U_{Q^{\prime}}$, we have $x \in \oplus_{\alpha \neq 0}$ Lie $G_{\alpha}$. Hence $x_{0}=0$ and $x \in$ Lie $U_{Q}$. Thus, Lie $Q \cap$ Lie $U_{Q^{\prime}} \subset$ Lie $U_{Q} \cap$ Lie $U_{Q^{\prime}}$. The reverse inclusion is obvious.

Lemma 8.7. Let $P, P^{\prime}, Q$ be parabolics such that $\operatorname{pos}\left(P^{\prime}, P\right)=z$. Assume that $\operatorname{pos}\left(P^{\prime}, Q\right)=y$, that $P^{\prime}, Q$ are in good position and that $Q$ contains a Levi of $P \cap P^{\prime}$. Let $Q^{\prime}=Q^{\left(P^{\prime P}\right)}$. Then

(a) $\operatorname{pos}\left(P^{P^{\prime}}, P^{\prime P}\right)=z^{-1}$.

(b) $\operatorname{pos}\left(P^{\prime P}, Q^{\prime}\right)=y$.

(c) $\operatorname{pos}\left(P^{P^{\prime}}, Q^{\prime}\right)=z^{-1} y$ and $P^{P^{\prime}}, Q^{\prime}$ are in good position.

(a) follows from 8.2; (b) follows from 8.3. We prove (c). Let $L_{0}$ be a common Levi of $P^{P^{\prime}}, P^{\prime P}$ that is contained in $Q$. Then $P^{P^{\prime}}, P^{\prime P}, Q^{\prime}$ have a common Levi $L_{0}$. By Lemma 8.4 we have $\operatorname{pos}\left(P^{P^{\prime}}, Q^{\prime}\right)=\operatorname{pos}\left(P^{P^{\prime}}, P^{\prime P}\right) \operatorname{pos}\left(P^{\prime P}, Q^{\prime}\right)=z^{-1} y$ as required.

8.8. Let $J, J^{\prime} \subset I$ and $y \in W$ be such that $\operatorname{Ad}(y) \delta(J)=J^{\prime}, y \in{ }^{J^{\prime}} W^{\delta(J)}$. For $P, P^{\prime}$ in $\mathcal{P}_{J}, \mathcal{P}_{J^{\prime}}$ let

$$
\begin{aligned}
& A_{y}\left(P, P^{\prime}\right)=\left\{g \in G^{1} ; \operatorname{pos}\left(P^{\prime},{ }^{g} P\right)=y\right\} \\
& A_{y}^{\prime}\left(P, P^{\prime}\right)=\left\{g \in A_{y}\left(P, P^{\prime}\right) ;{ }^{g} P \text { contains some Levi of } P \cap P^{\prime}\right\} .
\end{aligned}
$$

Lemma 8.9. (a) $A_{y}\left(P, P^{\prime}\right)$ is a single $P^{\prime}, P$ double coset and also a single $P^{\prime}, U_{P}$ double coset.

(b) $A_{y}\left(P, P^{\prime}\right)=U_{P^{\prime}} A_{y}^{\prime}\left(P, P^{\prime}\right)$.

(c) $A_{y}^{\prime}\left(P, P^{\prime}\right)$ is a single $\left(P \cap P^{\prime}\right), P$ double coset and also a single $U_{P \cap P^{\prime}}, P$ double coset.

We prove (a). We can find $Q \in \mathcal{P}_{\delta(J)}$ such that $\operatorname{pos}\left(P^{\prime}, Q\right)=y$; moreover, we can find $g \in G^{1}$ such that ${ }^{g} P=Q$. Thus $A_{y}\left(P, P^{\prime}\right) \neq \emptyset$. Let $g, g^{\prime} \in A_{y}\left(P, P^{\prime}\right)$. 
Clearly, $g^{\prime}=x g p$ with $x \in P^{\prime}, p \in P$. Now ${ }^{g} P, P^{\prime}$ are in good position; let $L$ be a common Levi of them. Since $g^{-1} L$ is a Levi of $P$, we have $p \in g^{-1} L U_{P}$. Thus $p=g^{-1} l g u$ with $l \in L, u \in U_{P}$ and $g^{\prime}=x g p=x l g u \in P^{\prime} g U_{P}$.

We prove (b). Let $g \in A_{y}\left(P, P^{\prime}\right)$. Let $L_{0}$ be a Levi of $P \cap P^{\prime}$. Then $L_{0}$ is contained in a Levi $L_{1}$ of $P^{\prime}$. Let $L_{2}$ be a common Levi of $P^{\prime},{ }^{g} P$. Then $L_{1}={ }^{u} L_{2}$ for some $u \in U_{P^{\prime}}$. We have ${ }^{u g} P={ }^{u}\left({ }^{g} P\right) \supset{ }^{u} L_{2}=L_{1} \supset L_{0}$ hence $u g \in A_{y}^{\prime}\left(P, P^{\prime}\right)$.

We prove (c). Let $g, g^{\prime} \in A_{y}^{\prime}\left(P, P^{\prime}\right)$. Then

$P^{P^{\prime}}, P^{\prime P},\left({ }^{g} P\right)^{\left(P^{\prime P}\right)}$ have a common Levi;

$P^{P^{\prime}}, P^{\prime P},\left(g^{\prime} P\right)^{\left(P^{\prime P}\right)}$ have a common Levi;

$\operatorname{pos}\left(P^{\prime P},\left({ }^{g} P\right)^{\left(P^{\prime P}\right)}\right)=\operatorname{pos}\left(P^{\prime P},\left(g^{\prime} P\right)^{\left(P^{\prime P}\right)}\right)=y$.

By Lemma 8.5, there exists $x \in G$ which conjugates

$$
P^{P^{\prime}}, P^{\prime P},\left({ }^{g} P\right)^{\left(P^{\prime P}\right)} \text { to } P^{P^{\prime}}, P^{\prime P},\left(g^{\prime} P\right)^{\left(P^{\prime P}\right)} \text {. }
$$

Then $x \in P^{P^{\prime}} \cap P^{\prime P}=P \cap P^{\prime}$ and $x$ conjugates ${ }^{g} P$ to ${ }^{g^{\prime}} P$, since ${ }^{g} P$ to $g^{\prime} P$ are parabolics of type $\delta(J)$ containing $\left({ }^{g} P\right)^{\left(P^{\prime P}\right)},\left(g^{\prime} P\right)^{\left(P^{\prime P}\right)}$. Hence $x g \in g^{\prime} P$ that is $g^{\prime} \in x g P$. Let $M$ be a Levi of $P \cap P^{\prime}$ with $M \subset{ }^{g} P$. We can write $x=v m$ with $v \in U_{P \cap P^{\prime}}, m \in M$. Then $g^{-1} m g \in P, x g P=v m g P=v g g^{-1} m g P=v g P$. The lemma is proved.

8.10. Let $P \in \mathcal{P}_{J}, P^{\prime} \in \mathcal{P}_{J^{\prime}}$ be such that $\operatorname{pos}\left(P^{\prime}, P\right)=z$. Let

$$
J_{1}=J \cap \delta^{-1} \operatorname{Ad}\left(y^{-1} z\right) J, J_{1}^{\prime}=J \cap \operatorname{Ad}\left(z^{-1} y\right) \delta(J) .
$$

Then $\operatorname{Ad}\left(z^{-1} y\right) \delta\left(J_{1}\right)=J_{1}^{\prime}$. Let $g \in A_{y}^{\prime}\left(P, P^{\prime}\right)$. We set

$$
P_{1}=g^{-1}\left({ }^{g} P\right)^{\left(P^{\prime P}\right)} g, \quad P_{1}^{\prime}=P^{P^{\prime}}, \quad\left(P_{1}, P_{1}^{\prime}\right)=\alpha\left(P, P^{\prime}, g\right) .
$$

We have $P_{1} \subset P, P_{1}^{\prime} \subset P$ and, by Lemma $8.7, P_{1}^{\prime},{ }^{g} P_{1}$ are in good position,

$$
P_{1} \in \mathcal{P}_{J_{1}}, P_{1}^{\prime} \in \mathcal{P}_{J_{1}^{\prime}}, \quad \operatorname{pos}\left(P_{1}^{\prime},{ }^{g} P_{1}\right)=z^{-1} y
$$

(We have also $\operatorname{pos}\left(P_{1}^{\prime}, P^{\prime P}\right)=z^{-1}, \operatorname{pos}\left(P^{\prime P},{ }^{g} P_{1}\right)=y$ ). Thus, $z^{-1} y \in J_{1}^{\prime} W^{\delta\left(J_{1}\right)}$ and $g \in A_{z^{-1} y}\left(P_{1}, P_{1}^{\prime}\right)$.

Lemma 8.11. Let $g, g^{\prime} \in A_{y}^{\prime}\left(P, P^{\prime}\right), u^{\prime} \in U_{P^{\prime}}, u \in U_{P}$ with $g^{\prime}=u^{\prime} g u$. Then

(a) $g^{\prime}=u_{1}^{\prime} g u_{1}$ with $u_{1}^{\prime} \in U_{P_{1}^{\prime}}, u_{1} \in U_{P_{1}}$;

(b) we have $\alpha\left(P, P^{\prime}, g^{\prime}\right)=\left(P_{1}, P_{1}^{\prime}\right)$.

We prove (a). Since $P_{1} \subset P$ we have $U_{P} \subset U_{P_{1}}$. Hence we may assume that $u=1$. By Lemma 8.9(c) we have $g^{\prime}=v g p$ with $v \in U_{P \cap P^{\prime}}, p \in P$. Thus $g^{\prime}=v g p=u^{\prime} g$. Hence $v^{-1} u^{\prime}=g p g^{-1}$. Now $v^{-1} u^{\prime} \in U_{P \cap P^{\prime}} U_{P^{\prime}} \subset U_{P^{\prime P}}$. Thus $v^{-1} u^{\prime} \in U_{P^{\prime P}} \cap{ }^{g} P$. We have

$$
U_{P^{\prime P}} \cap{ }^{g} P=U_{P^{\prime P}} \cap{ }^{g} P_{1}=U_{P^{\prime P}} \cap U_{g} P_{1}
$$


(In general, if $R, S$ are parabolics in good position and $R^{\prime} \subset R, S^{\prime}=S^{R^{\prime}}$, then $U_{R^{\prime}} \cap S=U_{R^{\prime}} \cap S^{\prime}$. Indeed, $S^{\prime}=\left(R^{\prime} \cap S\right) U_{S}$ hence $U_{R^{\prime}} \cap S \subset S^{\prime}$. Since $R^{\prime}, S^{\prime}$ are in good position, we have $U_{R^{\prime}} \cap S^{\prime}=U_{R^{\prime}} \cap U_{S^{\prime}}$ by Lemma 8.6.) Thus, $g_{p g}{ }^{-1}=v^{-1} u^{\prime} \in U_{g_{1}}$ so that $p \in U_{P_{1}}$. Now $v \in U_{P \cap P^{\prime}} \subset U_{P^{P^{\prime}}}=U_{P_{1}^{\prime}}$. We see that $g^{\prime}=v g p$ with $v \in U_{P_{1}^{\prime}}, p \in U_{P_{1}}$. This proves (a).

We prove (b). We must show that

$$
u^{-1} g^{-1} u^{\prime-1}\left({ }^{u^{\prime} g u} P\right)^{\left(P^{\prime P}\right)} u^{\prime} g u=g^{-1}\left({ }^{g} P\right)^{\left(P^{\prime P}\right)} g
$$

or that $u^{-1} g^{-1}\left({ }^{g} P\right)^{\left(P^{\prime P}\right)} g u=g^{-1}\left({ }^{g} P\right)^{\left(P^{\prime P}\right)} g$. This holds since $g^{-1}\left({ }^{g} P\right)^{\left(P^{\prime P}\right)} g$ is a parabolic subgroup of $P$ hence it contains $u$.

Lemma 8.12. Let $g, g^{\prime} \in A_{y}^{\prime}\left(P, P^{\prime}\right)$. Assume that $\alpha\left(P, P^{\prime}, g\right)=\alpha\left(P, P^{\prime}, g^{\prime}\right)=$ $\left(P_{1}, P_{1}^{\prime}\right)$ and that $g^{\prime} \in U_{P_{1}^{\prime}} g U_{P_{1}}$. Then there exist $x \in U_{P} \cap P^{\prime}, w^{\prime} \in U_{P^{\prime}}, w \in U_{P}$ such that $g^{\prime}=w^{\prime} x g w$.

By Lemma $8.9(\mathrm{c})$ we have $g^{\prime}=v g p$ with $v \in U_{P \cap P^{\prime}}, p \in P$. By assumption, $p^{-1} g^{-1} v^{-1}\left({ }^{v g p} P\right)^{\left(P^{\prime P}\right)} v g p=g^{-1}\left({ }^{g} P\right)^{\left(P^{\prime P}\right)} g$ that is ${ }^{p^{-1}} P_{1}=P_{1}$, or $p \in P_{1}$. Also $g^{\prime}=u^{\prime} g u$ with $u^{\prime} \in U_{P_{1}^{\prime}}, u \in U_{P_{1}}$. Thus, $g^{\prime}=v g p=u^{\prime} g u$. Setting $\pi=p u^{-1} \in P_{1}$ we have $v g \pi=u^{\prime} g$ and $v^{-1} u^{\prime}={ }^{g} \pi$. Now $v^{-1} u^{\prime} \in U_{P \cap P^{\prime}} U_{P^{P^{\prime}}}=U_{P^{P^{\prime}}}$. Thus $v^{-1} u^{\prime} \in U_{P^{P^{\prime}}} \cap{ }^{g} P_{1}$. Since $P^{P^{\prime}},{ }^{g} P_{1}$ are in good position, we have $v^{-1} u^{\prime} \in U_{P^{P^{\prime}}} \cap$ $U_{g} P_{1}$. Thus ${ }^{g} \pi \in U_{g} P_{1}$ and $\pi \in U_{P_{1}}$. Since $u \in U_{P_{1}}$, we have $p \in U_{P_{1}}$. Thus $g^{\prime} \in$ $U_{P \cap P^{\prime}} g U_{P_{1}}=U_{P \cap P^{\prime}} U_{g} P_{1} g$. Now ${ }^{g} P_{1}=\left({ }^{g} P\right)^{\left(P^{\prime P}\right)}$ hence $U_{g} P_{1}=\left({ }^{g} P \cap U_{P^{\prime P}}\right) U_{g} P$ so that

$$
\begin{aligned}
& g^{\prime} \in U_{P \cap P^{\prime}}\left({ }^{g} P \cap U_{P^{\prime P}}\right) U_{g} P=U_{P \cap P^{\prime}}\left({ }^{g} P \cap U_{P^{\prime P}}\right) g U_{P} \subset U_{P^{\prime P}} g U_{P} \\
& =U_{P^{\prime}}\left(U_{P} \cap P^{\prime}\right) g U_{P} .
\end{aligned}
$$

Thus, $g^{\prime}=w^{\prime} x g w$ with $w^{\prime} \in U_{P^{\prime}}, x \in P^{\prime} \cap U_{P}, w \in U_{P}$, as desired.

8.13. We fix $z \in{ }^{J^{\prime}} W^{J}$. Let $J_{1}=J \cap \delta^{-1} \operatorname{Ad}\left(y^{-1} z\right) J, J_{1}^{\prime}=J \cap \operatorname{Ad}\left(z^{-1} y\right) \delta(J)$, so that $\operatorname{Ad}\left(z^{-1} y\right) \delta\left(J_{1}\right)=J_{1}^{\prime}$. Let $Q, Q^{\prime}$ in $\mathcal{P}_{J_{1}}, \mathcal{P}_{J_{1}^{\prime}}$ be such that $\operatorname{pos}\left(Q^{\prime}, Q\right) \in W_{J}$. Let $\gamma_{1}$ be a $U_{Q^{\prime}}, U_{Q}$ double coset in $A_{z^{-1} y}\left(Q, Q^{\prime}\right)$. Let $F^{\prime}$ be the set of all $\left(P, P^{\prime}, g\right)$ with $P \in \mathcal{P}_{J}, P^{\prime} \in \mathcal{P}_{J^{\prime}}, \operatorname{pos}\left(P^{\prime}, P\right)=z, g \in A_{y}^{\prime}\left(P, P^{\prime}\right)$ such that $\alpha\left(P, P^{\prime}, g\right)=\left(Q, Q^{\prime}\right)$ and $g \in \gamma_{1}$. (Since $Q^{\prime} \subset P, P$ is uniquely determined.) Let $F$ be the set of all $\left(P, P^{\prime}, \gamma\right)$ with $P \in \mathcal{P}_{J}, P^{\prime} \in \mathcal{P}_{J^{\prime}}, \operatorname{pos}\left(P^{\prime}, P\right)=z, \gamma \in U_{P^{\prime}} \backslash A_{y}\left(P, P^{\prime}\right) / U_{P}$ such that for some/any $g \in \gamma \cap A_{y}^{\prime}\left(P, P^{\prime}\right)$ we have $\alpha\left(P, P^{\prime}, g\right)=\left(Q, Q^{\prime}\right)$ and $g \in \gamma_{1}$. (The equivalence of "some/any" follows from Lemma 8.11.) Again, $P$ is uniquely determined. The map $F^{\prime} \rightarrow F,\left(P, P^{\prime}, g\right) \mapsto\left(P, P^{\prime}, U_{P^{\prime}} g U_{P}\right)$ is surjective by Lemma 8.9(b).

Lemma 8.14. $F^{\prime}$ is non-empty.

Let $g \in \gamma_{1}$. Then $\operatorname{pos}\left(Q^{\prime},{ }^{g} Q\right)=z^{-1} y$. Define $P \in \mathcal{P}_{J}$ by $Q \subset P$. Since $\operatorname{pos}\left(Q^{\prime}, Q\right) \in W_{J}$, we have also $Q^{\prime} \subset P$. Let $\tilde{P}, P^{\prime}$ in $\mathcal{P}_{J}, \mathcal{P}_{J^{\prime}}$ with $\operatorname{pos}\left(P^{\prime}, \tilde{P}\right)=z$. 
By Lemma $8.9(\mathrm{c})$, we can find $\tilde{g} \in A_{y}^{\prime}\left(\tilde{P}, P^{\prime}\right)$. Now $\alpha\left(\tilde{P}, P^{\prime}, \tilde{g}\right)=\left(\tilde{Q}, \tilde{Q}^{\prime}\right)$ with $\tilde{Q} \in \mathcal{P}_{J_{1}}, \tilde{Q}^{\prime} \in \mathcal{P}_{J_{1}^{\prime}}$ contained in $\tilde{P}$ and $\operatorname{pos}\left(\tilde{Q}^{\prime}, \tilde{g} \tilde{Q}\right)=z^{-1} y$. We have $Q^{\prime}={ }^{h} \tilde{Q}^{\prime}$ for some $h \in G$. Replacing $\tilde{P}, P^{\prime}, \tilde{g}$ by ${ }^{h} \tilde{P},{ }^{h} P^{\prime}, h \tilde{g} h^{-1}$, we may assume that $\tilde{Q}^{\prime}=Q^{\prime}$. Then $\tilde{P} \in \mathcal{P}_{J}$ contains $Q^{\prime}$ hence $\tilde{P}=P$. Now $\tilde{Q}, Q$ are contained in $P$ and have the same type hence $\tilde{Q}={ }^{p} Q$ for some $p \in P$. We have ${ }^{\tilde{g} p} Q=\left({ }^{\tilde{g}} P\right)^{\left(P^{\prime P}\right)}$ and $\tilde{g} p \in A_{y}^{\prime}\left(P, P^{\prime}\right)$ hence $\alpha\left(P, P^{\prime}, \tilde{g} p\right)=\left(Q, Q^{\prime}\right)$. Since $\operatorname{pos}\left(Q^{\prime},{ }^{g} Q\right)=\operatorname{pos}\left(Q^{\prime},{ }^{\tilde{g} p} Q\right)$ and $Q^{\prime},{ }^{g} Q$ are in good position, we have $\tilde{g} p=u^{\prime} g q$ with $q \in Q, u^{\prime} \in U_{Q^{\prime}}$. Thus $u^{\prime} g q \in A_{y}^{\prime}\left(P, P^{\prime}\right)$ and $\alpha\left(P, P^{\prime}, u^{\prime} g q\right)=\left(Q, Q^{\prime}\right)$. We have $u^{\prime} g \in A_{y}^{\prime}\left(P, P^{\prime}\right)$ and $\alpha\left(P, P^{\prime}, u^{\prime} g\right)=\left(Q, Q^{\prime}\right)$ (we use that $q \in Q$ ). Since $u^{\prime} g \in \gamma_{1}$, we see that $F^{\prime} \neq \emptyset$.

Lemma 8.15. Let $\left(P, P^{\prime}, g\right) \in F^{\prime}$. Then $(u, v) \mapsto\left(P,{ }^{u} P^{\prime}, U_{u} P^{\prime} u v g U_{P}\right)$ is a well defined, surjective map $\kappa: U_{P} \times\left(U_{P} \cap P^{\prime}\right) \rightarrow F$.

Let $u \in U_{P}, v \in U_{P} \cap P^{\prime}$. Clearly, $\left(P,{ }^{u} P^{\prime}, u v g\right) \in F^{\prime}$. Hence $\kappa$ is well defined. We show that $\kappa$ is surjective. Let $\left(P, \tilde{P}^{\prime}, \gamma\right) \in F$. We can find $\tilde{g} \in \gamma$ such that $\left(P, \tilde{P}^{\prime}, \tilde{g}\right) \in F^{\prime}$. We have $g \in \gamma_{1}, \tilde{g} \in \gamma_{1}$ hence $\tilde{g} \in U_{Q^{\prime}} g U_{Q}$. Since $\operatorname{pos}\left(P^{\prime}, P\right)=$ $\operatorname{pos}\left(\tilde{P}^{\prime}, P\right)$, we have $\tilde{P}^{\prime}={ }^{p} P^{\prime}$ for some $p \in P$. Since $P^{P^{\prime}}=P^{\tilde{P}^{\prime}}=P^{\left({ }^{p} P^{\prime}\right)}=$ $p P^{P^{\prime}} p^{-1}$, we have $p \in P^{P^{\prime}}$. Thus, $p=u \pi$ with $\pi \in P \cap P^{\prime}, u \in U_{P}$. Since ${ }^{p} P^{\prime}={ }^{u} P^{\prime}$, we may assume that $p=u \in U_{P}$ so that $\tilde{P}^{\prime}={ }^{u} P^{\prime}$. Applying Lemma 8.12 to $\left(P,{ }^{u} P^{\prime}, u g\right),\left(P,{ }^{u} P^{\prime}, \tilde{g}\right) \in F^{\prime}$ (instead of $\left.\left(P, P^{\prime}, g\right),\left(P, P^{\prime}, g^{\prime}\right)\right)$ we see that $\tilde{g}=w^{\prime} x u g w$ for some $x \in U_{P} \cap{ }^{u} P^{\prime}, w^{\prime} \in U_{u} P^{\prime}, w \in U_{P}$. Let $v=u^{-1} x u \in U_{P} \cap P^{\prime}$. Then $\tilde{g}=w^{\prime} u v g w$. Thus, $\left(P, \tilde{P}^{\prime}, \gamma\right)=\kappa(u, v)$. The lemma is proved.

Lemma 8.16. In the setup of Lemma 8.15, the following two conditions for $(u, v),\left(u, v^{\prime}\right)$ in $U_{P} \times\left(U_{P} \cap P^{\prime}\right)$ are equivalent:

(i) $\kappa(u, v)=\kappa\left(u^{\prime}, v^{\prime}\right)$;

(ii) $u^{\prime}=u f, v^{\prime}=f^{-1} d v$ for some $f \in U_{P} \cap P^{\prime}, d \in U_{P} \cap U_{P^{\prime}}$.

Assume that (i) holds. We have ${ }^{u} P^{\prime}={ }^{u^{\prime}} P^{\prime}, u v g \in U_{u} P^{\prime} u^{\prime} v^{\prime} g U_{P}$. Thus $u^{\prime}=u f$ with $f \in P^{\prime}$ (hence $f \in U_{P} \cap P^{\prime}$ ) and $u v g \in u U_{P^{\prime}} f v^{\prime} g U_{P}$, that is, $v \in U_{P^{\prime}} f v^{\prime} U_{g_{P}}$, so that $v \in f v^{\prime} U_{P^{\prime}} U_{g}$. We show that

$$
P^{\prime} \cap U_{P^{\prime}} U_{g}=U_{P^{\prime}}
$$

Assume that $x \in P^{\prime} \cap U_{P^{\prime}} U_{g_{P}}$. We must show that $x \in U_{P^{\prime}}$. We have $u_{1} x \in U_{g_{P}}$ with $u_{1} \in U_{P^{\prime}}$. Let $x^{\prime}=u_{1} x \in P^{\prime}$. Then $x^{\prime} \in P^{\prime} \cap U_{g_{P}}=U_{P^{\prime}} \cap U_{g}$ (by Lemma 8.6, which is applicable since $P^{\prime},{ }^{g} P$ have a common Levi). Thus, $x^{\prime} \in U_{P^{\prime}}$ hence $x \in U_{P^{\prime}}$, as required.

Applying (a) to $v^{\prime-1} f^{-1} v \in P^{\prime} \cap U_{P^{\prime}} U_{g}$, we see that $v^{\prime-1} f^{-1} v \in U_{P^{\prime}}$, so that $v^{\prime}=f^{-1} d v$ for some $d \in U_{P^{\prime}}$. We have $d=f v^{\prime} v^{-1} \in U_{P} \cap P^{\prime}$. Hence $d \in U_{P} \cap U_{P^{\prime}}$. Thus, (ii) holds. The converse is immediate. The lemma is proved.

8.17. We consider a new group structure $(d, f) \bullet\left(d^{\prime}, f^{\prime}\right)=\left(f^{\prime} d f^{\prime-1} d^{\prime}, f^{\prime} f\right)$ on $\left(U_{P} \cap U_{P^{\prime}}\right) \times\left(U_{P} \cap P^{\prime}\right)$ and a new group structure $(u, v) \bullet\left(u^{\prime}, v^{\prime}\right)=\left(u^{\prime} u, v v^{\prime}\right)$ on $U_{P} \times\left(U_{P} \cap P^{\prime}\right)$. Then

$$
\theta:\left(U_{P} \cap U_{P^{\prime}}\right) \times\left(U_{P} \cap P^{\prime}\right) \rightarrow U_{P} \times\left(U_{P} \cap P^{\prime}\right), \quad(d, f) \mapsto\left(f, f^{-1} d\right),
$$


is an (injective) group homomorphism for these new group structures. We can reformulate condition (ii) in 8.16 as follows:

$$
\left(u^{\prime}, v^{\prime}\right)=\theta(x) \bullet(u, v) \text { for some } x \in\left(U_{P} \cap U_{P^{\prime}}\right) \times\left(U_{P} \cap P^{\prime}\right) .
$$

We see that $\kappa$ defines a bijection

$$
\theta\left(\left(\left(U_{P} \cap U_{P^{\prime}}\right) \times\left(U_{P} \cap P^{\prime}\right)\right) \backslash\left(U_{P} \times\left(U_{P} \cap P^{\prime}\right)\right)\right) \stackrel{\sim}{\longrightarrow} F
$$

One can check that this is in fact an isomorphism of algebraic varieties. Since $U_{P} \times\left(U_{P} \cap P^{\prime}\right)$ is a connected unipotent group and $\theta\left(\left(U_{P} \cap U_{P^{\prime}}\right) \times\left(U_{P} \cap P^{\prime}\right)\right)$ is a connected closed subgroup of it, we see that

(a) $F$ is isomorphic to an affine space of dimension $\operatorname{dim}\left(U_{P} /\left(U_{P} \cap U_{P^{\prime}}\right)\right)$.

8.18. Let $J, J^{\prime} \subset I$ and $y \in{ }^{J^{\prime}} W^{\delta(J)}$ be such that $\operatorname{Ad}(y) \delta(J)=J^{\prime}$. Let

$$
Z_{J, y, \delta}=\left\{\left(P, P^{\prime}, \gamma\right) ; P \in \mathcal{P}_{J}, P^{\prime} \in \mathcal{P}_{J^{\prime}}, \gamma \in U_{P^{\prime}} \backslash A_{y}\left(P, P^{\prime}\right) / U_{P}\right\}
$$

To any $\left(P, P^{\prime}, \gamma\right) \in Z_{J, y, \delta}$ we associate a sequence $\left(J_{n}, J_{n}^{\prime}, u_{n}\right)_{n \geq 0}$ with $J_{n}, J_{n}^{\prime} \subset$ $I, u_{n} \in W$, a sequence $\left(y_{n}\right)_{n \geq 0}$ with $y_{n} \in J_{n}^{\prime} W^{\delta\left(J_{n}\right)}, \operatorname{Ad}\left(y_{n}\right) \delta\left(J_{n}\right)=J_{n}^{\prime}$ and a sequence $\left(P_{n}, P_{n}^{\prime}, \gamma_{n}\right)_{n \geq 0}$ with $P_{n} \in \mathcal{P}_{J_{n}}, P_{n}^{\prime} \in \mathcal{P}_{J_{n}^{\prime}}, \gamma_{n} \subset A_{y_{n}}\left(P_{n}, P_{n}^{\prime}\right)$. We set

$$
P_{0}=P, P_{0}^{\prime}=P^{\prime}, \gamma_{0}=\gamma, J_{0}=J, J_{0}^{\prime}=J^{\prime}, u_{0}=\operatorname{pos}\left(P_{0}^{\prime}, P_{0}\right), y_{0}=y \text {. }
$$

Assume that $n \geq 1$, that $P_{m}, P_{m}^{\prime}, \gamma_{m}, J_{m}, J_{m}^{\prime}, u_{m}, y_{m}$ are already defined for $m<n$ and that $u_{m}=\operatorname{pos}\left(P_{m}^{\prime}, P_{m}\right), P_{m} \in \mathcal{P}_{J_{m}}, P_{m}^{\prime} \in \mathcal{P}_{J_{m}^{\prime}}$ for $m<n$. Let

$$
\begin{gathered}
J_{n}=J_{n-1} \cap \delta^{-1} \operatorname{Ad}\left(y_{n-1}^{-1} u_{n-1}\right) J_{n-1}, J_{n}^{\prime}=J_{n-1} \cap \operatorname{Ad}\left(u_{n-1}^{-1} y_{n-1}\right) \delta\left(J_{n-1}\right), \\
P_{n}=g_{n-1}^{-1}\left({ }^{g_{n-1}} P_{n-1}\right)^{\left(P_{n-1}^{\prime}{ }^{P_{n-1}}\right)} g_{n-1} \in \mathcal{P}_{J_{n}}, P_{n}^{\prime}=P_{n-1}^{P_{n-1}^{\prime}} \in \mathcal{P}_{J_{n}^{\prime}}
\end{gathered}
$$

where

$$
\begin{gathered}
g_{n-1} \in \gamma_{n-1} \cap A_{y_{n-1}}^{\prime}\left(P_{n-1}, P_{n-1}^{\prime}\right), \\
u_{n}=\operatorname{pos}\left(P_{n}^{\prime}, P_{n}\right), y_{n}=u_{n-1}^{-1} y_{n-1}, \gamma_{n}=U_{P_{n}^{\prime}} g_{n-1} U_{P_{n}} .
\end{gathered}
$$

This completes the inductive definition; the definition makes sense (it is independent of choices) by 8.9-8.11. We have $\left(J_{n}, J_{n}^{\prime}, u_{n}\right)_{n \geq 0} \in S(J, \operatorname{Ad}(y) \delta)$ (see 2.3). We write $\left(J_{n}, J_{n}^{\prime}, u_{n}\right)_{n \geq 0}=\beta\left(P, P^{\prime}, \gamma\right)$. For $\mathbf{s} \in S(J, \operatorname{Ad}(y) \delta)$ let

$$
Z_{J, y, \delta}^{\mathbf{s}}=\left\{\left(P, P^{\prime}, \gamma\right) \in Z_{J, y, \delta} ; \beta\left(P, P^{\prime}, \gamma\right)=\mathbf{s}\right\}
$$

Clearly, $\left(Z_{J, y, \delta}^{\mathbf{s}}\right)_{\mathbf{s} \in S(J, \operatorname{Ad}(y) \delta)}$ is a partition of $Z_{J, y, \delta}$ into locally closed subvarieties. The $G$-action on $Z_{J, y, \delta}$ given by $g:\left(P, P^{\prime}, \gamma\right) \mapsto\left({ }^{g} P,{ }^{g} P^{\prime}, g \gamma g^{-1}\right)$ preserves each of the pieces $Z_{J, y, \delta}^{\mathbf{s}}$. Now $\left(P, P^{\prime}, \gamma\right) \mapsto\left(P_{1}, P_{1}^{\prime}, \gamma_{1}\right)$ is a morphism $f: Z_{J, y, \delta}^{\mathbf{s}} \rightarrow$ $Z_{J_{1}, y_{1}, \delta}^{\mathbf{s}_{1}}$ where for $\mathbf{s}=\left(J_{n}, J_{n}^{\prime}, u_{n}\right)_{n \geq 0} \in S(J, \operatorname{Ad}(y) \delta)$ we set $\mathbf{s}^{1}=\left(J_{n}, J_{n}^{\prime}, u_{n}\right)_{n \geq 1} \in$ $S\left(J_{1}, \operatorname{Ad}\left(y_{1}\right) \delta\right)$. 
Lemma 8.19. (a) The morphism $\vartheta: Z_{J, y, \delta}^{\mathbf{s}} \rightarrow Z_{J_{1}, y_{1}, \delta}^{\mathbf{s}_{1}}$ is a locally trivial fibration with fibres isomorphic to an affine space of dimension $l\left(u_{0}\right)+\nu_{J}-\nu_{J_{1}}$.

(b) Let $\bar{\vartheta}$ be the map from the set of $G$-orbits on $Z_{J, y, \delta}^{\mathbf{s}}$ to the set of $G$-orbits on $Z_{J_{1}, y_{1}, \delta}^{\mathbf{s}_{1}}$ induced by $\vartheta$. Then $\bar{\vartheta}$ is a bijection.

We prove (a). Let $\left(P, P^{\prime}, \gamma\right) \in Z_{J, y, \delta}^{\mathrm{s}}$. From 8.17 and 8.1 (a) we see that each fibre of $\vartheta$ is an affine space of dimension

$\operatorname{dim}\left(U_{P} /\left(U_{P} \cap U_{P^{\prime}}\right)\right)=l(u)+\nu_{J}-\nu_{J \cap \operatorname{Ad}\left(u_{0}^{-1}\right) J^{\prime}}=l(u)+\nu_{J}-\nu_{J_{1}^{\prime}}=l(u)+\nu_{J}-\nu_{J_{1}}$.

The verification of local triviality is omitted.

We prove (b). From the fact that $\vartheta$ is surjective (see (a)) and $G$-equivariant, it follows that $\bar{\vartheta}$ is well defined and surjective. We show that $\bar{\vartheta}$ is injective. Let $\left(P, P^{\prime}, \gamma\right),\left(\tilde{P}, \tilde{P}^{\prime}, \tilde{\gamma}\right)$ be two triples in $Z_{J, y, \delta}^{\text {s }}$ whose images under $\vartheta$ are in the same $G$-orbit; we must show that these two triples are in the same $G$-orbit. Since $\vartheta$ is $G$ equivariant, we may assume that $\vartheta\left(P, P^{\prime}, \gamma\right)=\vartheta\left(\tilde{P}, \tilde{P}^{\prime}, \tilde{\gamma}\right)=\left(Q, Q^{\prime}, \gamma_{1}\right) \in Z_{J_{1}, y_{1}, \delta}^{\mathbf{s}_{1}}$. Define $F$ in terms of $\left(Q, Q^{\prime}, \gamma_{1}\right)$ as in 8.13. Then $\left(P, P^{\prime}, \gamma\right) \in F,\left(\tilde{P}, \tilde{P}^{\prime}, \tilde{\gamma}\right) \in F$. Since $P, \tilde{P}$ are parabolics of the same type containing $Q^{\prime}$ we have $P=\tilde{P}$. Let $g \in \gamma \cap A_{y}^{\prime}\left(P, P^{\prime}\right)$. By Lemma 8.15, there exist $u \in U_{P}, v \in U_{P} \cap P^{\prime}$ such that $\tilde{P}^{\prime}={ }^{u} P^{\prime}, \tilde{\gamma}=U_{u} P^{\prime} u v g U_{P}$. We have also $\tilde{P}={ }^{u v} P($ since $u v \in P), \tilde{P}^{\prime}={ }^{u v} P^{\prime}$ (since $v \in P^{\prime}$ ),

$$
\tilde{\gamma}=u U_{P^{\prime}} v g U_{P}=u v U_{P^{\prime}} g U_{P}=u v U_{P^{\prime}} g U_{P} v^{-1} u^{-1}=u v \gamma v^{-1} u^{-1}
$$

(since $v$ normalizes $U_{P^{\prime}}$ and $\left.u v \in U_{P}\right)$. Thus, $\left(\tilde{P}, \tilde{P}^{\prime}, \tilde{\gamma}\right)$ is obtained by the action of $u v \in G$ on $\left(P, P^{\prime}, \gamma\right)$, hence $\left(\tilde{P}, \tilde{P}^{\prime}, \tilde{\gamma}\right)$ is in the $G$-orbit of $\left(P, P^{\prime}, \gamma\right)$. The lemma is proved.

Lemma 8.20. Let $\mathbf{s}=\left(J_{n}, J_{n}^{\prime}, u_{n}\right)_{n \geq 0} \in S(J, \operatorname{Ad}(y) \delta)$. Then $Z_{J, y, \delta}^{\mathbf{s}}$ is an iterated affine space bundle (with fibre dimension $l(w)+\nu_{J}-\nu_{J_{m}}, w=u_{0} u_{1} \ldots u_{m}, m \gg 0$ ) over a fibre bundle over $\mathcal{P}_{J_{m}}$ with fibres isomorphic to $P / U_{P}$ with $P \in \mathcal{P}_{J_{m}}$, $m \gg 0$. In particular, $Z_{J, y, \delta}^{\mathbf{s}} \neq \emptyset$.

Assume first that $\mathbf{s}$ is such that $J_{n}=J_{n}^{\prime}=J$ and $u_{n}=1$ for all $n \geq 0$. (Then $\operatorname{Ad}(y) \delta(J)=J, y \in{ }^{J} W^{\delta(J)}$.) In this case, $Z_{J, y, \delta}^{\mathbf{s}}$ is the set of all $\left(P, P^{\prime}, \gamma\right)$ with $P=P^{\prime} \in \mathcal{P}_{J}, \gamma \in U_{P} \backslash A_{y}(P, P) / U_{P}$. (The associated sequence $\left(P_{n}, P_{n}^{\prime}, \gamma_{n}\right)$ is in this case $P_{n}=P_{n}^{\prime}=P, \gamma_{n}=\gamma$.) Thus, $Z_{J, y, \delta}^{\mathrm{s}}$ is a locally trivial fibration over $\mathcal{P}_{J}$ with fibres isomorphic to $P / U_{P}$ for $P \in \mathcal{P}_{J}$ and the lemma holds.

We now consider a general $\mathbf{s}$. For any $r \geq 0$ let

$$
\mathbf{s}_{r}=\left(J_{n}, J_{n}^{\prime}, u_{n}\right)_{n \geq r} \in S\left(J_{r}, \operatorname{Ad}\left(y_{r}\right) \delta\right)
$$

( $y_{r}$ as in 8.18). By 8.19(a) we have a sequence of affine space bundles

$$
Z_{J, y, \delta}^{\mathbf{s}} \rightarrow Z_{J_{1}, y_{1}, \delta}^{\mathbf{s}_{1}} \rightarrow Z_{J_{2}, y_{2}, \delta}^{\mathbf{s}_{2}} \rightarrow \ldots
$$


where for $r \gg 0, Z_{J_{r}, y_{r}, \delta}^{\mathbf{s}_{r}}$ is as in the first part of the proof. By 8.19(a), the sum of dimensions of fibres of the maps in this sequence is

$$
\sum_{n \geq 0}\left(l\left(u_{n}\right)+\nu_{J_{n}}-\nu_{J_{n+1}}\right)=\sum_{n \geq 0} l\left(u_{n}\right)+\nu_{J_{0}}-\nu_{J_{m}}=l(w)+\nu_{J}-\nu_{J_{m}}
$$

where $m \gg 0$. The lemma follows.

Remark. If $\hat{G}$ is defined over the finite field $\mathbf{F}_{p}$ and $\mathbf{k}$ is the algebraic closure of $\mathbf{F}_{p}$ then the number $N$ of rational points of $Z_{J, J^{\prime}, y}^{\mathbf{s}}$ over a sufficiently large finite subfield $\mathbf{F}_{q}$ of $\mathbf{k}$ equals

$$
\sharp\left(G\left(\mathbf{F}_{q}\right)\right) q^{l(w)+\nu_{J}-\nu_{I}} .
$$

Indeed, for $m \gg 0$ we have $N=\sharp\left(G\left(\mathbf{F}_{q}\right) / P_{m}\left(\mathbf{F}_{q}\right)\right) \frac{\sharp\left(P_{m}\left(\mathbf{F}_{q}\right)\right)}{q^{\nu_{I}-\nu_{m}}} q^{l(w)+\nu_{J}-\nu_{J_{m}}}$. Note that $l(w)+\nu_{J}-\nu_{I} \geq 0$.

8.21. In the setup of 8.20 , the maps in 8.20 (a) induce bijections on the sets of $G$-orbits (see $8.19(\mathrm{~b})$ ). Thus we obtain a canonical bijection between the set of $G$-orbits on $Z_{J, y, \delta}^{\mathbf{s}}$ and the set of $G$-orbits on $Z_{J_{r}, y_{r}, \delta}^{\mathbf{s}_{r}}$ with $r$ large enough so that $J_{r}=J_{r}^{\prime}=J_{r+1}=J_{r+1}^{\prime}=\ldots, u_{r}=u_{r+1}=\cdots=1$. This last set of orbits is canonically the set of $Q$-orbits on $U_{Q} \backslash A_{y_{r}}(Q, Q) / U_{Q}$ where $Q \in \mathcal{P}_{J_{r}}$. The $Q$-action (conjugation) factors through $Q / U_{Q}$. Let $L^{\mathbf{s}}$ be a Levi of $Q$. Then

$$
C^{\mathbf{s}}=\left\{g \in G^{1} ;{ }^{g} L^{\mathbf{s}}=L^{\mathbf{s}}, \operatorname{pos}\left({ }^{g} Q, Q\right)=y_{r}\right\}
$$

is a connected component of $N_{\hat{G}}\left(L^{\mathbf{s}}\right)$. We have an obvious bijection $C^{\mathbf{s}} \stackrel{\sim}{\longrightarrow}$ $U_{Q} \backslash A_{y_{r}}(Q, Q) / U_{Q}$ under which the action of $L^{\mathbf{s}}$ on $C^{\mathbf{s}}$ by conjugation corresponds to the action of $Q / U_{Q}$ on $U_{Q} \backslash A_{y_{r}}(Q, Q) / U_{Q}$ by conjugation. Thus we obtain a canonical bijection between the set of $G$-orbits on $Z_{J, y, \delta}^{\mathbf{s}}$ and the set of $L^{\mathbf{s}}$-conjugacy classes in $C^{\mathbf{s}}$ (a connected component of an algebraic group with identity component $L^{\mathbf{s}}$ ). Putting together these bijections we obtain a bijection

$$
G \backslash Z_{J, y, \delta} \leftrightarrow \sqcup_{\mathbf{s} \in S(J, \operatorname{Ad}(y) \delta)} L^{\mathbf{s}} \backslash C^{\mathbf{s}}
$$

where $G \backslash Z_{J, y, \delta}$ is the set of $G$-orbits on $Z_{J, y, \delta}$ and $L^{\mathbf{s}} \backslash C^{\mathbf{s}}$ is the set of $L^{\mathbf{s}}$-orbits on $C^{\mathbf{s}}$ (for the conjugation action).

\section{Comparison OF tWo PARTitions}

9.1. In the case where $y=1$, Sections 3 and 8 provide two methods to partition $Z_{J, y, \delta}$. In this section we show that the resulting partitions of $Z_{J, y, \delta}$ are the same. Lemmas 9.2, 9.3 hold for any $y$, but in $9.4,9.5$ we assume that $y=1$.

Lemma 9.2. Let $\left(P, P^{\prime}, \gamma\right) \in Z_{J, y, \delta}$. Let $n \geq 1$. Let $P_{1}^{\prime}, P_{n}$ be as in 8.18. We have $\operatorname{pos}\left(P^{\prime}, P_{n}\right)=\operatorname{pos}\left(P^{\prime}, P_{1}^{\prime}\right) \operatorname{pos}\left(P_{1}^{\prime}, P_{n}\right)$. 
Let $z=\operatorname{pos}\left(P^{\prime}, P_{1}^{\prime}\right), \tilde{z}=\operatorname{pos}\left(P^{\prime}, P_{n}\right), x=\operatorname{pos}\left(P_{1}^{\prime}, P_{n}\right)$. We have $z=\operatorname{pos}\left(P^{\prime}, P\right)$ hence $z \in{ }^{J^{\prime}} W^{J}$. We have also $\tilde{z} \in J^{\prime} W$. Since $P_{n} \subset P$ we have $\tilde{z} \in W_{J^{\prime}} z W_{J}$. Using 2.1(c) with $x, x^{\prime}$ replaced by $z, \tilde{z}$, we see that $\tilde{z}=z v$ with $v \in W_{J}$. Let $B, B^{\prime} \in \mathcal{B}$ be such that $B \subset P^{\prime}, B^{\prime} \subset P_{n}, \operatorname{pos}\left(B, B^{\prime}\right)=\tilde{z}$. Since $z \in W^{J}$, we have $l(z v)=l(z)+l(v)$. Hence there is a unique $B^{\prime \prime} \in \mathcal{B}$ such that $\operatorname{pos}\left(B, B^{\prime \prime}\right)=z$, $\operatorname{pos}\left(B^{\prime \prime}, B^{\prime}\right)=v$. Since $B^{\prime} \subset P$ and $\operatorname{pos}\left(B^{\prime \prime}, B\right) \in W_{J}$, we have $B^{\prime \prime} \subset P$. Since $B \subset P^{\prime}, B^{\prime \prime} \subset P$ and $\operatorname{pos}\left(B, B^{\prime \prime}\right)=\operatorname{pos}\left(P^{\prime}, P\right)=z$, we have $B^{\prime \prime} \subset P^{P^{\prime}}=P_{1}^{\prime}$. Since $B^{\prime \prime} \subset P_{1}^{\prime}, B^{\prime} \subset P_{n}$, we have $\operatorname{pos}\left(B^{\prime \prime}, B^{\prime}\right) \geq x$, hence $v \geq x$. We can find $B_{1}, B_{2} \in \mathcal{B}$ such that $B_{1} \subset P_{1}^{\prime}, B_{2} \subset P_{n}, \operatorname{pos}\left(B_{1}, B_{2}\right)=x$. Since $\operatorname{pos}\left(P^{\prime}, P\right)=z$ and $B_{1} \subset P^{P^{\prime}}$, we can find $B_{0} \in \mathcal{B}$ such that $B_{0} \subset P^{\prime}, \operatorname{pos}\left(B_{0}, B_{1}\right)=z$. Since $z \in W^{J}, x \in W_{J}$, we have $\operatorname{pos}\left(B_{0}, B_{2}\right)=z x$. We have $B_{0} \subset P^{\prime}, B_{2} \subset P_{n}$ hence $\operatorname{pos}\left(B_{0}, B_{2}\right) \geq \operatorname{pos}\left(P^{\prime}, P_{n}\right)$, that is, $z x \geq \tilde{z}=z v$. Thus, we have $v \geq x$ and $z x \geq z v$. Since $z \in W^{J}$ and $x, v \in W_{J}$ we have $x=v$. Thus, $\tilde{z}=z x$. The lemma is proved.

Lemma 9.3. Let $\left(P, P^{\prime}, \gamma\right) \in Z_{J, y, \delta}$. Let $P_{n}^{\prime}, P_{n}, u_{n}, \gamma_{n}$ be as in 8.18. For any $n \geq 0$ we have $\operatorname{pos}\left(P^{\prime}, P_{n}\right)=u_{0} u_{1} \ldots u_{n}$.

We argue by induction on $n=0$. For $n=0$ the result is clear. Assume now that $n \geq 1$. We have $\operatorname{pos}\left(P^{\prime}, P_{1}^{\prime}\right)=\operatorname{pos}\left(P^{\prime}, P^{P^{\prime}}\right)=\operatorname{pos}\left(P^{\prime}, P\right)=u_{0}$. Using the induction hypothesis for $\left(P_{1}, P_{1}^{\prime}, \gamma_{1}\right)$ and $n-1$ (instead of $\left(P, P^{\prime}, \gamma\right)$ and $n$ ) we see that $\operatorname{pos}\left(P_{1}^{\prime}, P_{n}\right)=u_{1} \ldots u_{n}$. By Lemma 9.2 we have $\operatorname{pos}\left(P^{\prime}, P_{n}\right)=$ $\operatorname{pos}\left(P^{\prime}, P_{1}^{\prime}\right) \operatorname{pos}\left(P_{1}^{\prime}, P_{n}\right)=u_{0}\left(u_{1} \ldots u_{n}\right)$. The lemma is proved.

Lemma 9.4. Let $J \subset I$. Let $\left(P, P^{\prime}, g\right) \in \mathcal{Z}_{J, \delta}$. To $\left(P, P^{\prime}, g\right)$ we associate $P^{n}, P^{\prime n}, w_{n}$ as in 4.11. To $\left(P, P^{\prime}, U_{P^{\prime}} g U_{P}\right)$ we associate $P_{n}, P_{n}^{\prime}, \gamma_{n}, u_{n}, y_{n}$ as in 8.18 (with $y=1)$. For any $n \geq 0$, the following hold:

$$
\begin{aligned}
& \text { (an) } P_{n}=P^{n} \text {; } \\
& \left(b_{n}\right) P_{n}^{\prime}=\left(P^{n-1}\right)^{P^{\prime n-1}} \text {; } \\
& \left(c_{n}\right) g \in \gamma_{n} \cap A_{y_{n}}^{\prime}\left(P_{n}, P_{n}^{\prime}\right) \text {; } \\
& \left(d_{n}\right)\left(P^{\prime n}\right)^{\left(P_{n}^{\prime P^{n}}\right)}=\left(P^{\prime n}\right)^{P^{n}} \text {; } \\
& \left(e_{n}\right) w_{n}=u_{0} u_{1} \ldots u_{n} \text {. }
\end{aligned}
$$

We argue by induction on $n$. The result is obvious for $n=0$ : we have $P_{0}=P^{0}=$ $P ;{ }^{g} P_{0}=P^{\prime}$ contains a Levi of $P \cap P^{\prime}$; we have $P^{\prime\left(P^{\prime P}\right)}=P^{\prime P}$. We have $w_{0}=u_{0}$. We now assume that $n \geq 1$ and that $\left(a_{n-1}\right),\left(b_{n-1}\right),\left(c_{n-1}\right),\left(d_{n-1}\right),\left(e_{n-1}\right)$ hold.

We show that $\left(a_{n}\right)$ holds. Using $\left(c_{n-1}\right),\left(a_{n-1}\right),\left(d_{n-1}\right)$, we have

$$
\begin{aligned}
& P_{n}=g^{-1}\left({ }^{g} P_{n-1}\right)^{\left(P_{n-1}^{\prime}{ }^{P_{n-1}}\right)} g=g^{-1}\left({ }^{g} P^{n-1}\right)^{\left(P_{n-1}^{\prime}{ }^{n-1}\right)} g \\
& =g^{-1}\left(P^{\prime n-1}\right)^{\left(P_{n-1}^{\prime}{ }^{n-1}\right)} g=g^{-1}\left(P^{\prime n-1}\right)^{P^{n-1}} g=P^{n} .
\end{aligned}
$$

hence $\left(a_{n}\right)$ holds.

We show that $\left(b_{n}\right)$ holds, using $\left(a_{n-1}\right),\left(b_{n-1}\right),\left(e_{n-1}\right),\left(a_{n}\right)$. It suffices to show that $Y^{\prime}=P_{n}^{\prime}$ satisfies the hypotheses of Lemma 3.2(a) with $P, P^{\prime}, a, J, \delta(J)$ replaced by $P^{n-1}, P^{\prime n-1}, w_{n-1}, J_{n-1}, \delta\left(J_{n-1}\right)$. By definition we have $Y^{\prime} \subset P_{n-1}=$ 
$P^{n-1}$. The type of $Y^{\prime}$ is $J_{n-1} \cap \operatorname{Ad}\left(u_{0} u_{1} \ldots u_{n-1}\right)^{-1} \delta\left(J_{n-1}\right)$ while that of $\left(P^{n-1}\right)^{P^{\prime n-1}}$ is $J_{n-1} \cap \operatorname{Ad}\left(w_{n-1}^{-1}\right) \delta\left(J_{n-1}\right)$. Thus, $Y^{\prime}$ and $\left(P^{n-1}\right)^{P^{\prime n-1}}$ have the same type. Since ${ }^{g} P_{n}={ }^{g} P^{n}=P^{\prime n}=\left(P^{\prime n-1}\right)^{P^{n-1}}$ and $P_{n}^{\prime},{ }^{g} P_{n}$ are in good position, $\operatorname{pos}\left(P_{n}^{\prime},{ }^{g} P_{n}\right)=\left(u_{0} u_{1} \ldots u_{n-1}\right)^{-1}=w_{n-1}^{-1}$, we see that $\left(P^{\prime n-1}\right)^{P^{n-1}}, P_{n}^{\prime}$ are in good position, $\operatorname{pos}\left(\left(P^{\prime n-1}\right)^{P^{n-1}}, P_{n}^{\prime}\right)=w_{n-1}$. Thus, the hypotheses of Lemma $3.2(\mathrm{a})$ are satisfied and $\left(b_{n}\right)$ holds.

We show that $\left(c_{n}\right)$ holds, using $\left(a_{n}\right),\left(b_{n}\right),\left(c_{n-1}\right) . \quad$ Since $g \in \gamma_{n-1} \cap A_{y_{n-1}}^{\prime}\left(P_{n-1}, P_{n-1}^{\prime}\right)$ and $\gamma_{n-1} \cap A_{y_{n-1}}^{\prime}\left(P_{n-1}, P_{n-1}^{\prime}\right) \subset \gamma_{n} \cap A_{y_{n}}\left(P_{n}, P_{n}^{\prime}\right)$ (see 8.10) we have $g \in \gamma_{n} \cap A_{y_{n}}\left(P_{n}, P_{n}^{\prime}\right)$. It remains to show that ${ }^{g} P_{n}$ contains a Levi of $P_{n} \cap P_{n}^{\prime}$ or equivalently, that $\left(P^{\prime n-1}\right)^{P^{n-1}}$ contains a Levi of $\left(P^{n-1}\right)^{P^{\prime n-1}} \cap g^{-1}\left(P^{\prime n-1}\right)^{P^{n-1}} g$. This follows from Lemma $3.2(\mathrm{~b})$ with $P, P^{\prime}, Z$ replaced by $P^{n-1}, P^{\prime n-1}, g^{-1}\left(P^{\prime n-1}\right)^{P^{n-1}} g$.

We show that $\left(d_{n}\right)$ holds, using $\left(a_{n}\right),\left(b_{n}\right)$. This follows from Lemma $3.2(\mathrm{~d})$ with $P, P^{\prime}, Z$ replaced by $P^{n-1}, P^{\prime n-1}, g^{-1}\left(P^{\prime n-1}\right)^{P^{n-1}} g$.

We show that $\left(e_{n}\right)$ holds, using $\left(a_{n}\right)$. Since $\operatorname{pos}\left(P^{\prime n}, P^{n}\right)=w_{n}, P^{\prime n} \subset P^{\prime}$ and $w_{n} \in{ }^{J} W$ we have $\operatorname{pos}\left(P^{\prime}, P^{n}\right)=w_{n}$. We also have $\operatorname{pos}\left(P^{\prime}, P_{n}\right)=u_{0} u_{1} \ldots u_{n}$ (see Lemma 9.3). Since $P^{n}=P_{n}$, we have $w_{n}=u_{0} u_{1} \ldots u_{n}$.

This completes the inductive proof.

Proposition 9.5. Let $J \subset I$. Let $\mathbf{s}=\left(J_{n}, J_{n}^{\prime}, u_{n}\right)_{n \geq 0} \in S(J, \delta)$ and let $\mathbf{t} \in \mathcal{T}(J, \delta)$ be the corresponding element under the bijection in 2.4. Then $Z_{J, \delta}^{\mathbf{s}}={ }^{\mathbf{t}} Z_{J, \delta}$.

Let $P \in \mathcal{P}_{J}, P^{\prime} \in \mathcal{P}_{\delta(J)}, g \in A_{1}\left(P, P^{\prime}\right)=A_{1}^{\prime}\left(P, P^{\prime}\right)$. Assume that

$$
\left(P, P^{\prime}, U_{P^{\prime}} g U_{P}\right) \in Z_{J, \delta}^{\tilde{\mathbf{s}}},\left(P, P^{\prime}, U_{P^{\prime}} g U_{P}\right) \in{ }^{\mathbf{t}} Z_{J, \delta}
$$

where $\tilde{\mathbf{s}}=\left(\tilde{J}_{n}, \tilde{J}_{n}^{\prime}, \tilde{u}_{n}\right)_{n \geq 0} \in S(J, \delta)$. Using Lemma 9.4(e), we see that

$$
\tilde{u}_{0} \tilde{u}_{1} \ldots \tilde{u}_{n}=u_{0} u_{1} \ldots u_{n}
$$

for all $n$. Using Lemma 2.5, we have $\tilde{\mathbf{s}}=\mathbf{s}$. Thus, ${ }^{\mathbf{t}} Z_{J, \delta} \subset Z_{J, \delta}^{\mathbf{s}}$. Conversely, let $\left(Q, Q^{\prime}, \gamma\right) \in Z_{J, \delta}^{\mathbf{s}}$. We have $\left(Q, Q^{\prime}, \gamma\right) \in \mathbf{t}^{\prime} Z_{J, \delta}$ for a unique $\mathbf{t}^{\prime} \in \mathcal{T}(J, \delta)$. By the first part of the proof we have $\left(Q, Q^{\prime}, \gamma\right) \in Z_{J, \delta}^{\mathbf{s}^{\prime}}$ where $\mathbf{s}^{\prime} \in S(J, \delta)$ corresponds to $\mathbf{t}^{\prime}$ under the bijection in 2.4. We have $\left(Q, Q^{\prime}, \gamma\right) \in Z_{J, \delta}^{\mathbf{s}} \cap Z_{J, \delta}^{\mathbf{s}^{\prime}}$ and the sets $Z_{J, \delta}^{\mathbf{s}}, Z_{J, \delta}^{\mathbf{s}^{\prime}}$ are either disjoint or coincide. Thus, $\mathbf{s}=\mathbf{s}^{\prime}$ hence $\mathbf{t}=\mathbf{t}^{\prime}$. We see that $Z_{J, \delta}^{\mathbf{s}} \subset{ }^{\mathbf{t}} Z_{J, \delta}$. The proposition is proved.

\section{EXAMPLE}

10.1. We consider an example. Let $V$ be a finite dimensional k-vector space. Let $G=\hat{G}=G^{1}=G L(V)$. Consider two $n$-step filtrations $V_{*}, V_{*}^{\prime}$ :

$$
0=V_{0} \subset V_{1} \subset V_{2} \subset \ldots \subset V_{n}=V, \quad 0 \subset V_{1}^{\prime} \subset V_{2}^{\prime} \subset \ldots \subset V_{n}^{\prime}=V
$$

of $V$. The type of $V_{*}$ is by definition the set

$$
J=\left\{i \in[1, n-1] ; \operatorname{dim} V_{k} \neq i \quad \forall k \in[0, n]\right\} .
$$


To $V_{*}, V_{*}^{\prime}$ we associate two $n^{2}$-step filtrations $X_{*}, X_{*}^{\prime}$ :

$$
\begin{aligned}
& 0=X_{10} \subset X_{11} \subset X_{12} \subset \ldots \subset X_{1 n}=X_{20} \subset X_{21} \subset X_{22} \subset X_{2 n}=X_{30} \\
& \subset \ldots \subset X_{n, n-1} \subset X_{n n}=V \\
& 0=X_{10}^{\prime} \subset X_{11}^{\prime} \subset X_{12}^{\prime} \subset \ldots \subset X_{1 n}^{\prime}=X_{20}^{\prime} \subset X_{21}^{\prime} \subset X_{22}^{\prime} \subset X_{2 n}^{\prime}=X_{30}^{\prime} \\
& \subset \ldots \subset X_{n, n-1}^{\prime} \subset X_{n n}^{\prime}=V
\end{aligned}
$$

where

$$
\begin{aligned}
& X_{i j}=V_{i-1}+\left(V_{i} \cap V_{j}^{\prime}\right),(i, j) \in[1, n] \times[0, n], \\
& X_{i j}^{\prime}=V_{i-1}^{\prime}+\left(V_{i}^{\prime} \cap V_{j}\right),(i, j) \in[1, n] \times[0, n] .
\end{aligned}
$$

Here the indexing set is $[1, n] \times[0, n]$ with the identifications $1 n=20, \ldots,(n-1) n=$ $n 0$. We have $X_{i 0}=V_{i-1}, X_{i 0}^{\prime}=V_{i-1}^{\prime}$ for $i \in[1, n]$. Hence $X_{*}$ (resp. $\left.X_{*}^{\prime}\right)$ is a refinement of $V_{*}$ (resp. $\left.V_{*}^{\prime}\right)$. If the stabilizer of $V_{*}$ (resp. $\left.V_{*}^{\prime}\right)$ is the parabolic $P$ (resp. $P^{\prime}$ ) then the stabilizer of $X_{*}$ (resp. $X_{*}^{\prime}$ ) in $G$ is the parabolic $P^{P^{\prime}}$ (resp. $\left.P^{\prime P}\right)$. By Zassenhaus' lemma, we have a canonical isomorphism

$$
t: X_{i j}^{\prime} / X_{i, j-1}^{\prime} \stackrel{\sim}{\longrightarrow} X_{j i} / X_{j, i-1} \text { for all }(i, j) \in[1, n] \times[1, n]
$$

Assume that we are given a permutation $\sigma:[1, n] \rightarrow[1, n]$ and vector space isomorphisms $\alpha_{i}: V_{i} / V_{i-1} \rightarrow V_{\sigma(i)}^{\prime} / V_{\sigma(i)-1}^{\prime}$ for $i \in[1, n]$. Define a third $n^{2}$-step filtration $Y_{*}$ (refining $\left.V_{*}\right)$ :

$$
\begin{aligned}
& 0=Y_{10} \subset Y_{11} \subset Y_{12} \subset \ldots \subset Y_{1 n}=Y_{20} \subset Y_{21} \subset Y_{22} \subset Y_{2 n}=Y_{30} \\
& \subset \ldots \subset Y_{n, n-1} \subset Y_{n n}=V
\end{aligned}
$$

$Y_{i 0}=V_{i-1}$ for $i \in[1, n]$,

$Y_{i j}$ is the subspace of $V_{i}$ containing $V_{i-1}$ such that $a_{i}$ carries the subspace $Y_{i j} / Y_{i 0}$ of $V_{i} / V_{i-1}$ onto the subspace $X_{\sigma(i), j}^{\prime} / X_{\sigma(i), 0}^{\prime}$ of $V_{\sigma(i)}^{\prime} / V_{\sigma(i)-1}^{\prime}$.

The composition

$$
Y_{i j} / Y_{i, j-1} \stackrel{\alpha_{i}}{\longrightarrow} X_{\sigma(i), j}^{\prime} / X_{\sigma(i), j-1}^{\prime} \stackrel{t}{\rightarrow} X_{j, \sigma(i)} / X_{j, \sigma(i)-1}
$$

is an isomorphism $b_{i j}$ for $(i, j) \in[1, n] \times[1, n]$. Define a permutation $\tau:[1, n] \times$ $[1, n] \rightarrow[1, n] \times[1, n]$ by $\tau(i, j)=(j, \sigma(i))$.

Let $\Sigma$ be the set of all quadruples $\left(V_{*}, V_{*}^{\prime}, \sigma, a_{i}\right)$ with $V_{*}, V_{*}^{\prime}$ as above (of prescribed types) and $\sigma, a_{i}$ are as above. Then $\Sigma$ may be identified with a set $Z_{J, y, 1}$ attached to $G=G L(V)$. Here $J, \operatorname{Ad}(y) J$ are the types of $V_{*}, V_{*}^{\prime}$. Let $\left(V_{*}, V_{*}^{\prime}, \sigma, a_{i}\right) \in \Sigma$. We define a sequence $\left(V_{*}^{m}, V_{*}^{\prime m}, \sigma^{m}, a_{i}^{m}\right)_{m \geq 0}$ of quadruples of the same kind as $\left(V_{*}, V_{*}^{\prime}, \sigma, a_{i}\right)$ as follows. Set $\left(V_{*}^{0}, V_{*}^{\prime 0}, \sigma^{0}, a_{i}^{0}\right)=\left(V_{*}, V_{*}^{\prime}, \sigma, a_{i}\right)$. Assume that $m \geq 1$ and that $\left(V_{*}^{m-1}, V_{*}^{\prime m-1}, \sigma^{m-1}, a_{i}^{m-1}\right)$ is already defined. Then 
$\left(V_{*}^{m}, V_{*}^{\prime m}, \sigma^{m}, a_{i}^{m}\right)$ is attached to $\left(V_{*}^{m-1}, V_{*}^{\prime m-1}, \sigma^{m-1}, a_{i}^{m-1}\right)$ in the same way as $\left(Y_{*}, X_{*}, \tau, b_{i j}\right)$ was attached to $\left(V_{*}, V_{*}^{\prime}, \sigma, a_{i}\right)$. Then $V_{*}^{m}, V_{*}^{\prime m}$ are $n^{2^{m}}$-step filtrations of $V$ and, for $m>0, V_{*}^{m}, V_{*}^{\prime m}$ are refinements of $V_{*}^{m-1}$. Let $J_{m}$ (resp. $J_{m}^{\prime}$ ) be the type of $V_{*}^{m}\left(\operatorname{resp} . V_{*}^{\prime m}\right)$. The set of all $\left(V_{*}, V_{*}^{\prime}, \sigma, a_{i}\right) \in \Sigma$ such that $J_{m}, J_{m}^{\prime}$ and the relative position of $V_{*}^{m}, V_{*}^{\prime m}$ is specified for each $m \geq 0$ is a locally closed subvariety of $\Sigma$. Thus we obtain a partition of $\Sigma$ which coincides with the partition $Z_{J, y, 1}=\sqcup_{\mathrm{s}} Z_{J, y, 1}^{\mathrm{s}}$.

10.2. Let $V$ be a $\mathbf{k}$-vector space of dimension $d \geq 2$. Let $\Sigma$ be the set of all quadruples $\left(V_{1}, V_{1}^{\prime}, a, b\right)$ where $V_{1}, V_{1}^{\prime}$ are lines in $V$, and $a: V_{1} \stackrel{\sim}{\longrightarrow} V_{1}^{\prime}, b: V / V_{1} \stackrel{\sim}{\longrightarrow}$ $V / V_{1}^{\prime}$ are isomorphisms. (This is a special case of the situation in 10.1 where we omit the 0 and $d$ dimensional members of a 2-step filtration.) We describe explicitly in this case the partition of $\Sigma$ given in 10.1 . For any $k \in[1, d]$ let $\tilde{\Sigma}_{k}$ be the set of all quadruples

$$
\left(0=V_{0} \subset V_{1} \subset V_{2} \subset \ldots \subset V_{k}, V_{1}^{\prime}, a, b\right)
$$

where $0=V_{0} \subset V_{1} \subset V_{2} \subset \ldots \subset V_{k}$ is a (partial) flag in $V$, $\operatorname{dim} V_{j}=j$ for all $j$, $V_{1}^{\prime}$ is a line in $V, a: V_{1} \stackrel{\sim}{\longrightarrow} V_{1}^{\prime}, b: V / V_{1} \stackrel{\sim}{\longrightarrow} V / V_{1}^{\prime}$ are isomorphisms and

$$
\begin{aligned}
& V_{1}^{\prime} \cap V_{k-1}=0, V_{1}^{\prime} \subset V_{k}, \\
& b\left(V_{j} / V_{1}\right)=\left(V_{j-1}+V_{1}^{\prime}\right) / V_{1}^{\prime} \text { for } j \in[1, k] .
\end{aligned}
$$

Define $\pi_{k}: \tilde{\Sigma}_{k} \rightarrow \Sigma$ by $\pi_{k}\left(0=V_{0} \subset V_{1} \subset V_{2} \subset \ldots \subset V_{k}, V_{1}^{\prime}, a, b\right)=\left(V_{1}, V_{1}^{\prime}, a, b\right)$. Then $\pi_{k}$ is injective and $\left(\pi_{k}\left(\Sigma_{k}\right)\right)_{k \in[1, d]}$ is a partition of $\Sigma$ into $d$ locally closed subvarieties. This is a special case of the partition of $\Sigma$ in 10.1 and of the partition of $Z_{J, y, \delta}$ in 8.18 .

10.3. Let $V$ be a $\mathbf{k}$-vector space of dimension $d \geq 2$. Let $\Sigma$ be the set of all quadruples $\left(V_{1}, H, a, b\right)$ where $V_{1}$ is a line in $V, H$ is a hyperplane in $V$, and $a: V_{1} \stackrel{\sim}{\rightarrow} V / H, b: V / V_{1} \stackrel{\sim}{\longrightarrow} H$ are isomorphisms. (This is a special case of the situation in 10.1 where we omit the 0 and $d$ dimensional members of a 2-step filtration.) We describe explicitly in this case the partition of $\Sigma$ given in 10.1. For any $k \in[1, d]$ let $\tilde{\Sigma}_{k}$ be the set of all quadruples

$$
\left(0=V_{0} \subset V_{1} \subset V_{2} \subset \ldots \subset V_{k}, H, a, b\right)
$$

where $0=V_{0} \subset V_{1} \subset V_{2} \subset \ldots \subset V_{k-1} \subset V_{k}$ is a (partial) flag in $V$, $\operatorname{dim} V_{j}=j$ for all $j, H$ is a hyperplane in $V, a: V_{1} \stackrel{\sim}{\longrightarrow} V / H, b: V / V_{1} \stackrel{\sim}{\longrightarrow} H$ are isomorphisms and

$$
\begin{aligned}
& V_{k-1}=V_{k} \cap H, \\
& b\left(V_{j} / V_{1}\right)=V_{j-1} \text { for } j \in[1, k] .
\end{aligned}
$$

Define $\pi_{k}: \tilde{\Sigma}_{k} \rightarrow \Sigma$ by $\pi_{k}\left(0=V_{0} \subset V_{1} \subset V_{2} \subset \ldots \subset V_{k}, H, a, b\right)=\left(V_{1}, H, a, b\right)$. Then $\pi_{k}$ is injective and $\left(\pi_{k}\left(\Sigma_{k}\right)\right)_{k \in[1, d]}$ is a partition of $\Sigma$ into $d$ locally closed subvarieties. This is a special case of the partition of $\Sigma$ in 10.1 and of the partition of $Z_{J, y, \delta}$ in 8.18 . 


\section{Parabolic character sheaves on $Z_{J, y, \delta}$}

11.1. Assume that we are in the setup of 8.18. Let $\mathbf{x}=\left(x_{1}, x_{2}, \ldots, x_{r}\right)$ be a sequence in $W$ such that

$$
r \geq 2, x_{r}=y \text {. }
$$

As in 4.2 we define

$Y_{\mathbf{x}}=\left\{\left(B_{0}, B_{1}, B_{2}, \ldots, B_{r}, g\right) \in \mathcal{B}^{r+1} \times G^{1} ; \operatorname{pos}\left(B_{i-1}, B_{i}\right)=x_{i}, i \in[1, r], B_{r}={ }^{g} B_{0}\right\}$.

Let ${ }^{y} Y_{\mathbf{x}}^{\prime}$ be the set of all $\left(B_{0}, B_{1}, B_{2}, \ldots, B_{r-1}, \gamma\right)$ where $\left(B_{0}, B_{1}, B_{2}, \ldots, B_{r-1}\right) \in$ $\mathcal{B}^{r}$ satisfies $\operatorname{pos}\left(B_{i-1}, B_{i}\right)=x_{i}, i \in[1, r-1]$ and $\gamma \in U_{P^{\prime}} \backslash A_{y}\left(P, P^{\prime}\right) / U_{P}$ (with $P \in \mathcal{P}_{J}, P^{\prime} \in \mathcal{P}_{J^{\prime}}$ given by $\left.B_{0} \subset P, B_{r-1} \subset P^{\prime}\right)$ satisfies $\operatorname{pos}\left(B_{r-1},{ }^{g} B_{0}\right)=x_{r}$ for some/any $g \in \gamma$. (This definition is correct since $U_{P} \subset B_{0}, U_{P^{\prime}} \subset B_{r-1}$.) We have an affine space bundle

$$
\omega: Y_{\mathbf{x}} \rightarrow{ }^{y} Y_{\mathbf{x}}^{\prime}, \quad\left(B_{0}, B_{1}, B_{2}, \ldots, B_{r}, g\right) \mapsto\left(B_{0}, B_{1}, B_{2}, \ldots, B_{r-1}, U_{P^{\prime}} g U_{P}\right)
$$

(with $P, P^{\prime}$ as above). Define

$$
\Pi_{\mathbf{x}}:{ }^{y} Y_{\mathbf{x}}^{\prime} \rightarrow Z_{J, y, \delta}, \quad \Pi_{\mathbf{x}}\left(B_{0}, B_{1}, \ldots, B_{r-1}, \gamma\right)=\left(P, P^{\prime}, \gamma\right)
$$

where $P \in \mathcal{P}_{J}, P^{\prime} \in \mathcal{P}_{J^{\prime}}$ are given by $B_{0} \subset P, B_{r-1} \subset P^{\prime}$.

If $\mathcal{L} \in \mathcal{S}(T)$ (see 4.1) is such that $x_{1} x_{2} \ldots x_{r} \in W_{\mathcal{L}}^{1}$ (see 4.1 ), the local system $\tilde{\mathcal{L}}$ on $Y_{\mathbf{x}}$ is defined (see 4.2 ); it is $\omega^{*}$ of a well defined local system on ${ }^{y} Y_{\mathbf{x}}^{\prime}$ denoted again by $\tilde{\mathcal{L}}$. We set

$$
\mathcal{K}_{\mathbf{x}}^{\mathcal{L}}=\left(\Pi_{\mathbf{x}}\right) ! \tilde{\mathcal{L}} \in \mathcal{D}\left(Z_{J, y, \delta}\right)
$$

Now assume in addition that

$$
x_{i} \in I \sqcup\{1\} \text { for } i \in[1, r-1] .
$$

Let $Y_{\mathbf{x}}^{\prime \dagger}$ be the set of all $\left(B_{0}, B_{1}, B_{2}, \ldots, B_{r-1}, \gamma\right)$ where $\left(B_{0}, B_{1}, B_{2}, \ldots, B_{r-1}\right) \in$ $\mathcal{B}^{r}$ satisfies $\operatorname{pos}\left(B_{i-1}, B_{i}\right)=\left\{x_{i}, 1\right\}, i \in[1, r-1]$ and $\gamma \in U_{P^{\prime}} \backslash A_{y}\left(P, P^{\prime}\right) / U_{P}$ (with $P \in \mathcal{P}_{J}, P^{\prime} \in \mathcal{P}_{J^{\prime}}$ given by $\left.B_{0} \subset P, B_{r-1} \subset P^{\prime}\right)$ satisfies $\operatorname{pos}\left(B_{r-1},{ }^{g} B_{0}\right)=y$ for some/any $g \in \gamma$. Define

$$
\Pi_{\mathbf{x}}^{\dagger}: Y_{\mathbf{x}}^{\prime \dagger} \rightarrow Z_{J, y, \delta} \quad \Pi_{\mathbf{x}}^{\dagger}\left(B_{0}, B_{1}, \ldots, B_{r-1}, \gamma\right)=\left(P, P^{\prime}, \gamma\right)
$$

where $P \in \mathcal{P}_{J}, P^{\prime} \in \mathcal{P}_{J^{\prime}}$ are given by $B_{0} \subset P, B_{r-1} \subset P^{\prime}$. Now ${ }^{y} Y_{\mathbf{x}}^{\prime}$ is an open dense subset subset of $Y_{\mathrm{x}}^{\prime \dagger}$ and it carries the local system $\tilde{\mathcal{L}}$. The intersection cohomology complex $I C\left(Y_{\mathbf{x}}^{\prime \dagger}, \tilde{\mathcal{L}}\right)$ is a constructible sheaf $\overline{\mathcal{L}}$ on $Y_{\mathbf{x}}^{\prime \dagger}$ (compare 4.3); we set

$$
\overline{\mathcal{K}}_{\mathbf{x}}^{\mathcal{L}}=\left(\Pi_{\mathbf{x}}^{\dagger}\right) ! \overline{\mathcal{L}} \in \mathcal{D}\left(Z_{J, y, \delta}\right) .
$$

Now $\Pi_{\mathbf{x}}^{\dagger}$ is a proper morphism. (Indeed, the condition $\operatorname{pos}\left(B_{r-1},{ }^{g} B_{0}\right)=y$ in the definition of $Y_{\mathrm{x}}^{\prime \dagger}$ can be replaced by the closed condition $\operatorname{pos}\left(B_{r-1},{ }^{g} B_{0}\right) \leq y$ since ${ }^{g} B_{0} \subset{ }^{g} P, B_{r-1} \subset P^{\prime}$ and $\operatorname{pos}\left(P^{\prime},{ }^{g} P\right)=y$.) Hence we may apply the decomposition theorem $[\mathrm{BBD}]$ and we see that

$\overline{\mathcal{K}}_{\mathbf{x}}^{\mathcal{L}}$ is a semisimple complex on $Z_{J, y, \delta}$. 
Proposition 11.2. Let $\mathcal{L} \in \mathcal{S}(T)$ and let $A$ be a simple perverse sheaf on $Z_{J, \delta}$. The following conditions on $A$ are equivalent:

(i) $A \dashv \mathcal{K}_{\mathbf{x}}^{\mathcal{L}}$ for some $\mathbf{x}$ as in 11.1(a) with $x_{1} x_{2} \ldots x_{r} \in W_{\mathcal{L}}^{1}$;

(ii) $A \dashv \mathcal{K}_{x, y}^{\mathcal{L}}$ for some $x \in W$ such that $x y \in W_{\mathcal{L}}^{1}$;

(iii) $A \dashv \overline{\mathcal{K}}_{\mathbf{x}}^{\mathcal{L}}$ for some $\mathbf{x}$ as in 11.1(b) with $x_{1} x_{2} \ldots x_{r} \in W_{\mathcal{L}}^{1}$.

(Compare 4.4.)

11.3. Let $\mathcal{C}_{J, y, \delta}^{\mathcal{L}}$ be the set of isomorphism classes of simple perverse sheaves on $Z_{J, y, \delta}$ which satisfy the equivalent conditions $11.2(\mathrm{i})$-(iii) with respect to $\mathcal{L}$. The simple perverse sheaves on $Z_{J, y, \delta}$ which belong to $\mathcal{C}_{J, y, \delta}^{\mathcal{L}}$ for some $\mathcal{L} \in \mathcal{T}$ are called parabolic character sheaves; they (or their isomorphism classes) form a set $\mathcal{C}_{J, y, \delta}$.

11.4. Let $\left(P, P^{\prime}, \gamma\right) \in Z_{J, y, \delta}$. Let $\gamma^{\prime}=\gamma \cap A_{y}^{\prime}\left(P, P^{\prime}\right)$. Let $z=\operatorname{pos}\left(P^{\prime}, P\right)$. Let $\left(P_{1}, P_{1}^{\prime}\right)=\alpha\left(P, P^{\prime}, g\right)$ where $g \in \gamma^{\prime}$ (see 8.10, 8.11). We have $\operatorname{pos}\left(P_{1}^{\prime},{ }^{g} P_{1}\right)=z^{-1} y$ and $\operatorname{pos}\left(P^{\prime P}, P^{P^{\prime}}\right)=z($ see 8.10$)$. Let $w \in W$. We can write uniquely $w=a b, a \in$ $W^{J^{\prime}}, b \in W_{J^{\prime}}$. Let

$$
\mathcal{X}=\left\{\left(B, B^{\prime}\right) \in \mathcal{B} \times \mathcal{B} ; \operatorname{pos}\left(B, B^{\prime}\right)=w, \operatorname{pos}\left(B^{\prime},{ }^{g} B\right)=y, B \subset P, B^{\prime} \subset P^{\prime}\right\}
$$

Here $g \in \gamma^{\prime}$; the choice of $g$ is irrelevant since $U_{P} \subset B, U_{P^{\prime}} \subset B^{\prime}$. Set $b^{\prime}=y^{-1} b y \in$ $W_{\delta(J)} \cdot\left(\right.$ Recall that $y W_{\delta(J)}=W_{J^{\prime}} y$.) Let

$$
\begin{aligned}
\mathcal{X}^{\prime}= & \left\{\left(\tilde{B}, B, \tilde{B}^{\prime}\right) \in \mathcal{B}^{3} ; \operatorname{pos}(\tilde{B}, B)=\delta^{-1}\left(b^{\prime}\right), \operatorname{pos}\left(B, \tilde{B}^{\prime}\right)=a z,\right. \\
& \left.\operatorname{pos}\left(\tilde{B}^{\prime},{ }^{g} \tilde{B}\right)=z^{-1} y, \tilde{B} \subset P_{1}, \tilde{B}^{\prime} \subset P_{1}^{\prime}\right\}
\end{aligned}
$$

(We have automatically $B \subset P$.) Here $g \in \gamma^{\prime}$; the choice of $g$ is irrelevant since $U_{P_{1}^{\prime}} \subset \tilde{B}^{\prime}, U_{P_{1}} \subset \tilde{B}$ (another choice of $g$ is in $U_{P_{1}^{\prime}} g U_{P_{1}}$ by 8.11). Define

$$
\mathcal{X} \stackrel{\mu}{\rightarrow} \mathcal{X}^{\prime}, \quad\left(B, B^{\prime}\right) \mapsto\left(\tilde{B}, B, \tilde{B}^{\prime}\right)
$$

as follows. Define $R$ by $\operatorname{pos}(B, R)=a, \operatorname{pos}\left(R, B^{\prime}\right)=b$ (we have $\left.l(w)=l(a)+l(b)\right)$. Define $\tilde{B}^{\prime}$ by $\operatorname{pos}\left(B, \tilde{B}^{\prime}\right)=a z, \operatorname{pos}\left(\tilde{B}^{\prime}, R\right)=z^{-1}$ (we have $\left.l(a z)+l\left(z^{-1}\right)=l(a)\right)$. Define $S_{g}$ (in terms of $\left.g \in \gamma^{\prime}\right)$ by $\operatorname{pos}\left(R, S_{g}\right)=y, \operatorname{pos}\left(S_{g},{ }^{g} B\right)=b^{\prime}$; we use $l(b)+$ $l(y)=l(b y)=l\left(y b^{\prime}\right)=l(y)+l\left(b^{\prime}\right)$. Set $\tilde{B}=g^{-1} S_{g}$. We will show below that

(i) if $\left(B, B^{\prime}\right) \in \mathcal{X}$ then $\left(\tilde{B}, B, \tilde{B}^{\prime}\right) \in \mathcal{X}^{\prime}$;

(ii) $\tilde{B}$ is independent of the choice of $g$ in $\gamma^{\prime}$.

Assume that (i) is already established (for fixed $g \in \gamma^{\prime}$ ). Let us replace $g$ by $g_{1} \in \gamma^{\prime}$. Then $g_{1}=u^{\prime} g u$ where $u \in U_{P}, u^{\prime} \in U_{P^{\prime}}$. We have $\operatorname{pos}\left(R, S_{g_{1}}\right)=$ $y, \operatorname{pos}\left(S_{g_{1}},{ }^{u^{\prime} g u} B\right)=b^{\prime}$. Hence

$$
\operatorname{pos}\left({ }^{u^{\prime-1}} R,{ }^{u^{\prime-1}} S_{g_{1}}\right)=y, \operatorname{pos}\left({ }^{u^{\prime-1}} S_{g_{1}},{ }^{g} B\right)=b^{\prime} .
$$


Since $\operatorname{pos}\left(R, B^{\prime}\right) \in W_{J^{\prime}}$ we have $R \subset P^{\prime}$ hence $u^{\prime} \in R$ and ${ }^{u^{\prime-1}} R=R$ and $\operatorname{pos}\left(R,{ }^{u^{\prime-1}} S_{g_{1}}\right)=y, \operatorname{pos}\left({ }^{u^{\prime-1}} S_{g_{1}},{ }^{g} B\right)=b^{\prime}$. It follows that $S_{g}={ }^{u^{\prime-1}} S_{g_{1}}$. Hence

$$
{ }^{u^{-1} g^{-1} u^{\prime-1}} S_{g_{1}}={ }^{u^{-1} g^{-1} u^{-1}}\left(u^{\prime} S_{g}\right)={ }^{u^{-1} g^{-1}} S_{g}={ }^{u^{-1}} \tilde{B}=\tilde{B}
$$

(since by (i), we have $\tilde{B} \subset P_{1} \subset P$ hence $u \in \tilde{B}$ ). Thus (ii) is verified.

We now verify (i) (with fixed $g \in \gamma^{\prime}$ ). Since $P^{P^{\prime}}, P^{\prime P},{ }^{g} P_{1}$ have a common Levi $L_{0}$, there is a canonical trijection ${ }^{0} B \leftrightarrow{ }^{1} B \leftrightarrow{ }^{2} B$ between the sets of Borels of $P^{P^{\prime}}, P^{\prime P},{ }^{g} P_{1}$ respectively, defined by ${ }^{0} B=U_{P^{P^{\prime}}} \beta,{ }^{1} B=U_{P^{\prime P}} \beta,{ }^{2} B=U_{g_{P_{1}}} \beta$ where $\beta$ is a Borel of $L_{0}$ or equivalently by

$$
\operatorname{pos}\left({ }^{0} B,{ }^{1} B\right)=z^{-1}, \operatorname{pos}\left({ }^{1} B,{ }^{2} B\right)=y, \operatorname{pos}\left({ }^{0} B,{ }^{2} B\right)=z^{-1} y
$$

(any two of these three conditions implies the third).

Assume that $B, B^{\prime}, R, S, t B, \tilde{B}^{\prime}$ are as above. We have $R \subset P^{\prime P}$ (since $a=$ $\left.\operatorname{pos}(B, R)=\operatorname{pos}\left(B, P^{\prime}\right)\right)$ and $\tilde{B}^{\prime} \subset P^{P^{\prime}}\left(\right.$ since $\left.z^{-1}=\operatorname{pos}\left(\tilde{B}^{\prime}, R\right)=\operatorname{pos}\left(P, P^{\prime}\right)\right)$. Hence $\tilde{B}^{\prime} \leftrightarrow R \leftrightarrow Y$ under the trijection above where $Y$ is a Borel of ${ }^{g} P_{1}$. (We have $\left.\operatorname{pos}\left(\tilde{B}^{\prime}, R\right)=z^{-1}, \operatorname{pos}(R, Y)=y, \operatorname{pos}\left(\tilde{B}^{\prime}, Y\right)=z^{-1} y.\right)$

Since $P^{\prime}, g P$ have a common Levi, there is a canonical bijection $B^{0} \leftrightarrow B^{1}$ between the sets of Borels of $P^{\prime},{ }^{g} P$ respectively, defined by $\operatorname{pos}\left(B^{0}, B^{1}\right)=y$. Since $\operatorname{pos}(R, Y)=y$, we have $R \leftrightarrow Y$ under this bijection; since $\operatorname{pos}(R, S)=y$, we have $R \leftrightarrow S$ under this bijection; hence $Y=S$. We see that $S \subset{ }^{g} P_{1}$ and $\tilde{B} \subset P_{1}$. We have $\operatorname{pos}\left(S,{ }^{g} B\right)=b^{\prime}$ hence $\operatorname{pos}(\tilde{B}, B)=\delta^{-1}\left(b^{\prime}\right)$. Define

$$
\mathcal{X}^{\prime} \stackrel{\mu^{\prime}}{\rightarrow} \mathcal{X}, \quad\left(\tilde{B}, B, \tilde{B}^{\prime}\right) \mapsto\left(B, B^{\prime}\right)
$$

as follows. Choose $g \in \gamma^{\prime}$. Define $R, S$ by the condition that $\tilde{B}^{\prime} \leftrightarrow R \leftrightarrow S$ under the canonical trijection above. Define $B^{\prime}$ by the condition $\operatorname{pos}\left(R, B^{\prime}\right)=$ $b, \operatorname{pos}\left(B^{\prime},{ }^{g} B\right)=y\left(\right.$ we use $\left.l(b)+l(y)=l(b y)=l\left(y b^{\prime}\right)=l(y)+l\left(b^{\prime}\right)\right)$. We will show below that

(iii) if $\left(\tilde{B}, B, \tilde{B}^{\prime}\right) \in \mathcal{X}^{\prime}$ then $\left(B, B^{\prime}\right) \in \mathcal{X}$;

(iv) $B^{\prime}$ is independent of the choice of $g$ in $\gamma^{\prime}$.

Now (iv) follows from (iii) in the same way that (ii) follows from (i). We now verify (iii) (with fixed $g \in \gamma^{\prime}$ ). Assume that $\tilde{B}, B, \tilde{B}^{\prime}, R, B^{\prime}$ are as above. We have $R \subset P^{\prime P}$ hence $R \subset P^{\prime}$. Since $\operatorname{pos}\left(R, B^{\prime}\right) \in W_{J^{\prime}}$ we have $B^{\prime} \subset P^{\prime}$. We have $\operatorname{pos}\left(B, \tilde{B}^{\prime}\right)=a z, \operatorname{pos}\left(\tilde{B}^{\prime}, R\right)=z^{-1}, l(a z)+l\left(z^{-1}\right)=l(a)$ hence $\operatorname{pos}(B, R)=a$. This, together with $\operatorname{pos}\left(R, B^{\prime}\right)=b, l(a b)=l(a)+l(b)$ implies $\operatorname{pos}\left(B, B^{\prime}\right)=a b=w$. Thus $\left(B, B^{\prime}\right) \in \mathcal{X}$ and $\mu^{\prime}$ is well defined.

From the definitions we see that $\mu^{\prime}$ is the inverse of $\mu$.

11.5. For $z \in{ }^{J^{\prime}} W^{J}$ let $Z_{J, y, \delta ; z}=\left\{\left(P, P^{\prime}, \gamma\right) \in Z_{J, y, \delta} ; \operatorname{pos}\left(P^{\prime}, P\right)=z\right\}$. As in $8.10,8.11$ we have a well defined map

$$
Z_{J, y, \delta ; z} \rightarrow Z_{J_{1}, z^{-1} y, \delta}, \quad\left(P, P^{\prime}, \gamma\right) \mapsto\left(P^{1}, P^{\prime 1}, \gamma_{1}\right)
$$


where $\gamma_{1}$ is given by $\gamma_{1} \subset \gamma \cap A_{y}^{\prime}\left(P, P^{\prime}\right)$. Let ${ }^{y} Y_{w, y}^{\prime}{ }^{z}$ be the inverse image of $Z J, y, \delta ; z$ under the canonical map ${ }^{y} Y_{w, y}^{\prime} \rightarrow Z_{J, y, \delta}$. For $a, b^{\prime}$ as in 11.4 , let

$$
Y_{\delta^{-1}\left(b^{\prime}\right), a z, z^{-1} y}^{\prime \prime}={ }^{-1} y Y_{\delta^{-1}\left(b^{\prime}\right), a z, z^{-1} y}^{\prime} \times_{Z_{J_{1}, z^{-1} y, \delta}} Z_{J, y, \delta ; z}
$$

where the fibre product is formed using the canonical maps

$$
{ }^{z^{-1} y} Y_{\delta^{-1}\left(b^{\prime}\right), a z, z^{-1} y}^{\prime} \rightarrow Z_{J_{1}, z^{-1} y, \delta} \leftarrow Z_{J, y, \delta ; z}
$$

The results in 11.4 provide an isomorphism

$$
{ }^{y} Y_{w, y}^{\prime} \stackrel{\sim}{\longrightarrow} Y_{\delta^{-1}\left(b^{\prime}\right), a z, z^{-1} y}^{\prime \prime}
$$

compatible with the natural maps of the two sides into $Z_{J, y, \delta ; z}$. Hence in the cartesian diagram

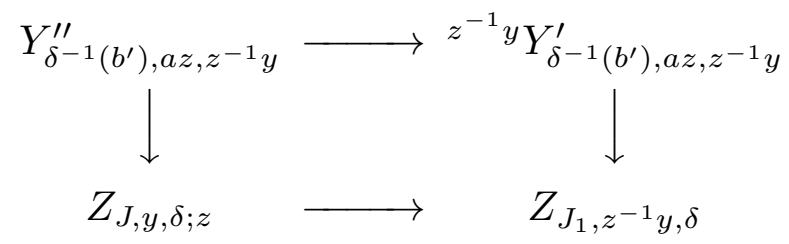

we may substitute $Y_{\delta^{-1}\left(b^{\prime}\right), a z, z^{-1} y}$ by ${ }^{y} Y_{w, y}^{\prime}{ }^{z}$ and we obtain a cartesian diagram

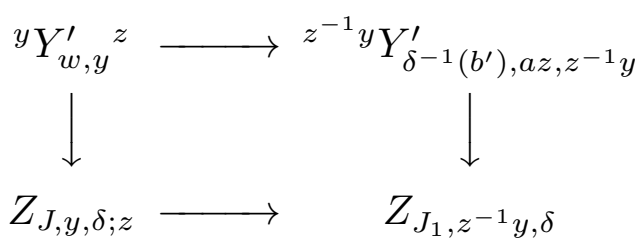

11.6. In the setup of 11.5 , let $\mathcal{L}, \mathcal{L}^{\prime} \in \mathcal{S}(T)$ be such that

$$
\mathcal{L}^{\prime}=\operatorname{Ad}\left(\delta^{-1}\left(b^{\prime-1}\right)\right)^{*} \mathcal{L}
$$

(we have $\left.\delta^{-1}\left(b^{\prime-1}\right) \in W_{J}\right)$ and

$$
w y \in W_{\mathcal{L}}^{1}, \quad \delta^{-1}\left(b^{\prime}\right) a z\left(z^{-1} y\right) \in W_{\mathcal{L}^{\prime}}^{1}
$$

(These two conditions are equivalent. In general, for $v \in W$ we have $W_{\operatorname{Ad}(v) * \mathcal{L}}^{1}=$ $v^{-1} W_{\mathcal{L}^{1}} \delta(v)$. In our case we have

$\left.\delta^{-1}\left(b^{\prime}\right) a z\left(z^{-1} y\right)=\delta^{-1}\left(b^{\prime}\right)\left(a y b^{\prime}\right) b^{\prime-1}=\delta^{-1}\left(b^{\prime}\right) w y b^{\prime-1}.\right)$

Let $\tilde{\mathcal{L}}, \tilde{\mathcal{L}}^{\prime}$ the local systems on ${ }^{y} Y_{w, y}^{\prime},{ }^{z^{-1} y} Y_{\delta^{-1}\left(b^{\prime}\right), a z, z^{-1} y}^{\prime}$ corresponding as in 11.1 to $\mathcal{L}, \mathcal{L}^{\prime}$. From the definitions we see that the inverse image of $\tilde{\mathcal{L}}^{\prime}$ under ${ }^{y} Y_{w, y}^{\prime}{ }^{z} \rightarrow$ ${ }^{z^{-1} y} Y_{\delta^{-1}\left(b^{\prime}\right), a z, z^{-1} y}^{\prime}$ (in the cartesian diagram above) is the same as the restriction of $\tilde{\mathcal{L}}$ to ${ }^{y} Y_{w, y}^{\prime}{ }^{z}$. 
11.7. In the setup of 11.4 , we assume in addition that $b \in W_{\operatorname{Ad}(y) \delta\left(J_{1}\right)}$ that is, $b^{\prime} \in W_{\delta\left(J_{1}\right)}$ where $J_{1}=J \cap \delta^{-1} \operatorname{Ad}\left(y^{-1} z\right) J$. Assume that $\left(B, B^{\prime}\right) \in \mathcal{X}$. We show that

$$
B \subset P_{1} \text {. }
$$

Let $R, S, \tilde{B}, \tilde{B}^{\prime}$ be as in the definition of $\mu$ in terms of some $g \in \gamma^{\prime}$. (Thus, $\mu\left(B, B^{\prime}\right)=\left(\tilde{B}, B, \tilde{B}^{\prime}\right)$.) By 11.4 , we have $S \subset{ }^{g} P_{1}$. Since $\operatorname{pos}\left(S,{ }^{g} B\right)=b^{\prime} \in W_{\delta\left(J_{1}\right)}$ and ${ }^{g} P_{1}$ has type $\delta\left(J_{1}\right)$, we have ${ }^{g} B \subset{ }^{g} P_{1}$ and $B \subset P_{1}$.

11.8. In the setup of 11.4 and 11.7 , we have for $B, B^{\prime}, \tilde{B}, \tilde{B}^{\prime}$ as in $11.7 a z=$ $\operatorname{pos}\left(B, \tilde{B}^{\prime}\right) \in{ }^{J_{1}} W\left(\right.$ since $\left.B \subset P_{1}\right)$ and $\operatorname{pos}(\tilde{B}, B)=\delta^{-1}\left(b^{\prime}\right) \in W_{J_{1}}$ hence

$$
l\left(\delta^{-1}\left(b^{\prime}\right) a z\right)=l\left(\delta^{-1}\left(b^{\prime}\right)\right)+l(a z) .
$$

It follows that $\operatorname{pos}\left(\tilde{B}, \tilde{B}^{\prime}\right)=\delta^{-1}\left(b^{\prime}\right) a z$. We see that $\left(\tilde{B}, B, \tilde{B}^{\prime}\right) \mapsto\left(\tilde{B}, \tilde{B}^{\prime}\right)$ defines an isomorphism $\mathcal{X}^{\prime} \stackrel{\sim}{\longrightarrow} \mathcal{X}^{\prime \prime}$ where

$\mathcal{X}^{\prime \prime}=\left\{\left(\tilde{B}, \tilde{B}^{\prime}\right) \in \mathcal{B}^{2} ; \operatorname{pos}\left(\tilde{B}, \tilde{B}^{\prime}\right)=\delta^{-1}\left(b^{\prime}\right) a z, \operatorname{pos}\left(\tilde{B}^{\prime},{ }^{g} \tilde{B}\right)=z^{-1} y, \tilde{B} \subset P_{1}, \tilde{B}^{\prime} \subset P_{1}^{\prime}\right\}$

Combining with the isomorphism $\mu: \mathcal{X} \stackrel{\sim}{\rightarrow} \mathcal{X}^{\prime}$ we see that $\left(B, B^{\prime}\right) \mapsto\left(t B, \tilde{B}^{\prime}\right)$ is an isomorphism

$$
\mathcal{X} \stackrel{\sim}{\longrightarrow} \mathcal{X}^{\prime \prime}
$$

Now let

$$
Y_{\delta^{-1}\left(b^{\prime}\right) a z, z^{-1} y}^{\prime \prime}={ }^{z^{-1} y} Y_{\delta^{-1}\left(b^{\prime}\right) a z, z^{-1} y}^{\prime} \times_{Z_{J_{1}, z^{-1} y, \delta}} Z_{J, y, \delta ; z}
$$

where the fibre product is formed using the canonical maps

$$
{ }^{z^{-1} y} Y_{\delta^{-1}\left(b^{\prime}\right) a z, z^{-1} y}^{\prime} \rightarrow Z_{J_{1}, z^{-1} y, \delta} \leftarrow Z_{J, y, \delta ; z}
$$

Then (a) gives rise to an isomorphism

$$
{ }^{y} Y_{w, y}^{\prime z} \stackrel{\sim}{\longrightarrow} Y_{\delta^{-1}}^{\prime \prime}\left(b^{\prime}\right) a z, z^{-1} y
$$

compatible with the natural maps of the two sides into $Z_{J, y, \delta ; z}$. As in 11.5 this gives rise to a cartesian diagram

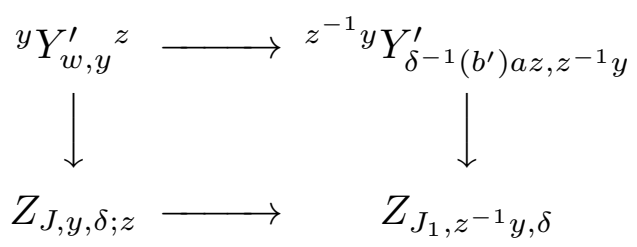

Now let $\mathcal{L}, \mathcal{L}^{\prime}$ be as in 11.6. Let $\tilde{\mathcal{L}}, \tilde{\mathcal{L}}^{\prime}$ the local systems on ${ }^{y} Y_{w, y}^{\prime},{ }^{-1} y Y_{\delta^{-1}}^{\prime}\left(b^{\prime}\right) a z, z^{-1} y$ corresponding as in 11.1 to $\mathcal{L}, \mathcal{L}^{\prime}$. From the definitions we see that the inverse image of $\tilde{\mathcal{L}}^{\prime}$ under ${ }^{y} Y_{w, y}^{\prime}{ }^{z} \rightarrow{ }^{z^{-1} y} Y_{\delta^{-1}\left(b^{\prime}\right) a z, z^{-1} y}^{\prime}$ (in the cartesian diagram above) is the same as the restriction of $\tilde{\mathcal{L}}$ to ${ }^{y} Y_{w, y}^{\prime}$. 
11.9. Let $w=a b, b^{\prime}$ be as in 11.4. Let $\mathbf{s}=\left(J_{n}, J_{n}^{\prime}, u_{n}\right)_{n \geq 0} \in S(J, \operatorname{Ad}(y) \delta)$. For $\mathbf{x}$ as in 11.1(a) let ${ }^{y} Y_{\mathbf{x}}^{\prime \mathbf{s}}$ be the inverse image of $Z J, y, \delta^{\mathbf{s}}$ under the canonical map ${ }^{y} Y_{\mathbf{x}}^{\prime} \rightarrow Z_{J, y, \delta}$. In the last (cartesian) diagram in 11.5 (with $z=u_{0}$ ), the inverse image of $Z_{J_{1}, z^{-1} y, \delta}^{\mathbf{s}_{1}}$

-under $Z_{J, y, \delta ; z} \rightarrow Z_{J_{1}, z^{-1} y, \delta}$ is $Z_{J, y, \delta}^{\mathrm{s}}$,

-under ${ }^{z^{-1} y} Y_{\delta^{-1}\left(b^{\prime}\right), a z, z^{-1} y}^{\prime} \rightarrow Z_{J_{1}, z^{-1} y, \delta}^{\mathbf{s}_{1}}$ is ${ }^{z^{-1} y} Y_{\delta^{-1}\left(b^{\prime}\right), a z, z^{-1} y}^{\prime} \mathbf{s}_{1}$,

-under ${ }^{y} Y_{w, y}^{\prime}{ }^{z} \rightarrow Z_{J_{1}, z^{-1} y, \delta}$ (the two compositions in the diagram) is ${ }^{y} Y_{w, y}^{\prime}{ }^{\mathbf{s}}$.

Therefore that cartesian diagram restricts to a cartesian diagram

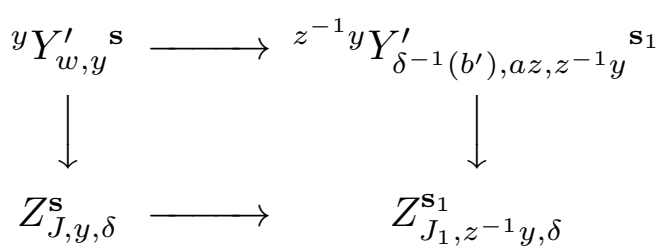

11.10. In the setup of 11.9 assume in addition that $b, b^{\prime}$ are as in 11.7. As in 11.9, the cartesian diagram in 11.8 restricts to a cartesian diagram

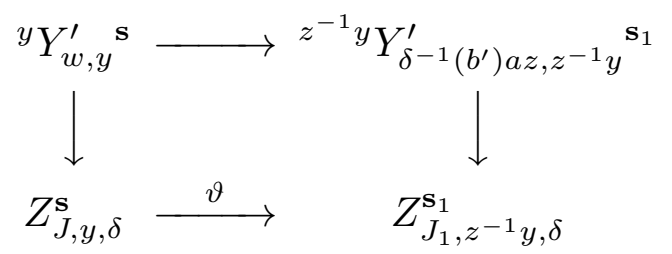

11.11. Let $\mathcal{L} \in \mathcal{S}(T)$, let $\mathbf{x}$ be as in 11.1 (a) (with $r=3$ ) and let $\mathbf{s} \in S(J, \operatorname{Ad}(y) \delta)$. Let $\mathcal{L} \in \mathcal{S}(T)$ be such that $x_{1} x_{2} y \in W_{\mathcal{L}}^{1}$ and let $\tilde{\mathcal{L}}$ be the corresponding local system on ${ }^{y} Y_{x_{1}, x_{2}, y}^{\prime}$. Let $A^{\prime}$ be a simple perverse sheaf on ${ }^{y} Y_{x_{1}, x_{2}, y}^{\prime}$ such that $\left.A^{\prime} \dashv\left(\left(\Pi_{x_{1}, x_{2}, y}\right) ! \tilde{\mathcal{L}}\right)\right|_{Z_{J, y, \delta}^{\text {s }}}$. We show that

(a) there exists $x_{0} \in W$ such that $x_{0} y \in W_{\mathcal{L}}^{1}$ and $\left.A^{\prime} \dashv\left(\left(\Pi_{x_{0}, y}\right)\right.$ ! $\left.\tilde{\mathcal{L}}\right)\right|_{Z_{J, y, \delta}^{\mathrm{s}}}$ (we denote the local system on ${ }^{y} Y_{x_{0}, y}^{\prime}$ corresponding to $\mathcal{L}$ again by $\tilde{\mathcal{L}}$ ).

The proof is similar to that of Lemma 4.8. We argue by induction on $l\left(x_{2}\right)$. If $l\left(x_{2}\right)=0$ then $x_{2}=1$, we may identify ${ }^{y} Y_{x_{1}, x_{2}, y}^{\prime},{ }^{y} Y_{x_{1}, y}^{\prime}$ and the result is obvious. Assume now that $l\left(x_{2}\right)>0$. We can find $s \in W$ such that $l(s)=1, l\left(x_{2}\right)>l\left(s x_{2}\right)$.

Assume first that $l\left(x_{1} s\right)=l\left(x_{1}\right)+1$. Then

$$
{ }^{y} Y_{x_{1}, x_{2}, y}^{\prime} \stackrel{\sim}{\rightarrow}{ }^{y} Y_{x_{1} s, s x_{2}, y}^{\prime}, \quad\left(B_{0}, B_{1}, B_{2}, \gamma\right) \mapsto\left(B_{0}, B_{1}^{\prime}, B_{2}, \gamma\right)
$$

with $B_{1}^{\prime}$ given by

$$
\operatorname{pos}\left(B_{1}, B_{1}^{\prime}\right)=s, \operatorname{pos}\left(B_{1}^{\prime}, B_{2}\right)=s x_{2}
$$

is an isomorphism. Under this isomorphism, $\tilde{\mathcal{L}}$ on ${ }^{y} Y_{x_{1}, x_{2}, y}^{\prime}$ corresponds to the analogous local system on ${ }^{y} Y_{x_{1} s, s x_{2}, y}^{\prime}$. We have $\left.A^{\prime} \dashv\left(\left(\Pi_{x_{1} s, s x_{2}, y}\right), \tilde{\mathcal{L}}\right)\right|_{Z_{J, y, \delta}^{\mathrm{s}}}$. We may apply the induction hypothesis to $x_{1} s, s x_{2}, y$; the desired result follows. 
Assume next that $l\left(x_{1} s\right)=l\left(x_{1}\right)+1$. Then we have a partition ${ }^{y} Y_{x_{1}, x_{2}, y}^{\prime}=$ ${ }^{\prime} Y \sqcup^{\prime \prime} Y$ where ${ }^{\prime} Y$ is the open subset defined by $\operatorname{pos}\left(B_{0}, B_{1}^{\prime}\right)=x_{1}$ (with $B_{1}^{\prime}$ as in (b)) and " $Y$ is the closed subset defined by $\operatorname{pos}\left(B_{0}, B_{1}^{\prime}\right)=x_{1} s$ (with $B_{1}^{\prime}$ as in (b)). Let ' $\Pi:{ }^{\prime} Y \rightarrow Z_{J, y, \delta},{ }^{\prime \prime} \Pi:{ }^{\prime \prime} Y \rightarrow Z_{J, y, \delta}$ be the restrictions of $\Pi_{x_{1}, x_{2}, y}$ to ${ }^{\prime} Y,{ }^{\prime \prime} Y$. By general principles, we have either

(c) $\left.A^{\prime} \dashv\left({ }^{\prime} \Pi_{!} \tilde{\mathcal{L}}\right)\right|_{Z_{J, y, \delta}^{\mathrm{s}}}$ or

(d) $\left.A^{\prime} \dashv\left({ }^{\prime \prime} \Pi_{!} \tilde{\mathcal{L}}\right)\right|_{Z_{J, y, \delta}^{\text {s }}}$

where the restriction of $\tilde{\mathcal{L}}$ to ${ }^{\prime} Y$ or ${ }^{\prime \prime} Y$ is denoted again by $\tilde{\mathcal{L}}$.

Assume that (d) holds. Then

$$
\iota^{\prime \prime}:{ }^{\prime \prime} Y \rightarrow{ }^{y} Y_{x_{1} s, s x_{2}, y}^{\prime}, \quad\left(B_{0}, B_{1}, B_{2}, \gamma\right) \mapsto\left(B_{0}, B_{1}^{\prime}, B_{2}, \gamma\right)
$$

with $B_{1}^{\prime}$ as in (b), is a line bundle and $\iota_{!}^{\prime \prime} \tilde{\mathcal{L}}$ is up to shift the local system on ${ }^{y} Y_{x_{1} s, s x_{2}, y}^{\prime}$ attached to $\mathcal{L}$ (we denote it again by $\tilde{\mathcal{L}}$ ). Since ${ }^{\prime \prime} \Pi=\Pi_{x_{1} s, s x_{2}, y} \iota^{\prime \prime}$, it follows that $\left.A^{\prime} \dashv\left(\left(\Pi_{x_{1} s, s x_{2}, y}\right) ! \tilde{\mathcal{L}}\right)\right|_{Z_{J, y, \delta}^{\mathrm{s}}}$. We may apply the induction hypothesis to $x_{1} s, s x_{2}, y$; the desired result follows.

Assume now that (c) holds. Then

$$
\iota^{\prime}:{ }^{\prime} Y \rightarrow{ }^{y} Y_{x_{1}, s x_{2}, y}^{\prime}, \quad\left(B_{0}, B_{1}, B_{2}, \gamma\right) \mapsto\left(B_{0}, B_{1}^{\prime}, B_{2}, \gamma\right)
$$

with $B_{1}^{\prime}$ as in (b), makes $Y^{\prime}$ into the complement of the zero section of a line bundle over ${ }^{y} Y_{x_{1}, s x_{2}, y}^{\prime}$ and we have ' $\Pi=\Pi_{x_{1} s, s x_{2}, y} \iota^{\prime}$. In the case where

(e) the inverse image of $\mathcal{L}$ under the coroot $\mathbf{k}^{*} \rightarrow T$ corresponding to $y^{-1} x_{2}^{-1} s x_{2} y$ is $\overline{\mathbf{Q}}_{l}$,

(so that $x_{1} s x_{2} y \in W_{\mathcal{L}}^{1}$ ), $\tilde{\mathcal{L}}$ (on ' $Y$ ) is $\iota^{\prime *}$ of the local system on ${ }^{y} Y_{x_{1}, s x_{2}, y}^{\prime}$ (denoted by $\left.\tilde{\mathcal{L}}^{\prime}\right)$ hence we have an exact triangle consisting of $\iota_{!}^{\prime} \tilde{\mathcal{L}}, \tilde{\mathcal{L}}^{\prime}$ and a shift of $\tilde{\mathcal{L}}^{\prime}$. Hence $\left.A^{\prime} \dashv\left(\left(\Pi_{x_{1}, s x_{2}, y}\right), \tilde{\mathcal{L}}\right)\right|_{Z_{J, y, \delta}^{\mathrm{s}}}$. We may apply the induction hypothesis to $x_{1}, s x_{2}, y$; the desired result follows. In the case where (e) does not hold, we have $\iota_{!}^{\prime} \tilde{\mathcal{L}}=0$ hence ${ }^{\prime} \Pi_{!} \tilde{\mathcal{L}}=0$, a contradiction. (a) is proved.

11.12. Let $\mathbf{s}=\left(J_{n}, J_{n}^{\prime}, u_{n}\right)_{n \geq 0} \in S(J, \operatorname{Ad}(y) \delta)$. For $r \gg 0$ we have $J_{r}=J_{r}^{\prime}=$ $J_{r+1}=J_{r+1}^{\prime}=\ldots$, and $u_{r}=u_{r+1}=\cdots=1$. Let $P \in \mathcal{P}_{J_{r}}$. Let $L^{\mathbf{s}}$ be a Levi of $P$. Then

$$
C^{\mathbf{s}}=\left\{g \in G^{1} ;{ }^{g} L^{\mathbf{s}}=L^{\mathbf{s}}, \operatorname{pos}\left(P,{ }^{g} P\right)=y_{r}\right\}
$$

(where $y_{r}=u_{r-1}^{-1} \ldots u_{0}^{-1} y$ ) is a connected component of $N_{\hat{G}}\left(L^{\mathbf{s}}\right)$. Let $X$ be a character sheaf on $C^{\mathbf{s}}$ (the definition in 4.5 is applicable since $C^{\mathbf{s}}$ is a connected component of an algebraic group with identity component $L^{\mathbf{s}}$ ). We regard $X$ as a simple perverse sheaf on $U_{P} \backslash A_{y_{r}}(P, P) / U_{P}$ via the obvious isomorphism $C^{\mathbf{s}} \stackrel{\sim}{\longrightarrow}$ $U_{P} \backslash A_{y_{r}}(P, P) / U_{P}$. Now $X$ is $P$-equivariant for the conjugation action of $P$. Hence there is a well defined simple perverse sheaf $X^{\prime}$ on $G \times_{P}\left(U_{P} \backslash A_{y_{r}}(P, P) / U_{P}\right)$ (with $P$ acting on $G$ by right translation) whose inverse image under

$$
G \times\left(U_{P} \backslash A_{y_{r}}(P, P) / U_{P}\right) \rightarrow G \times_{P}\left(U_{P} \backslash A_{y_{r}}(P, P) / U_{P}\right)
$$


is a shift of the inverse image of $X$ under

$$
p r_{2}: G \times\left(U_{P} \backslash A_{y_{r}}(P, P) / U_{P}\right) \rightarrow U_{P} \backslash A_{y_{r}}(P, P) / U_{P}
$$

We may regard $X^{\prime}$ as a simple perverse sheaf on $Z_{J_{r}, y_{r}, \delta}^{\mathbf{s}_{r}}$ via the isomorphism

$$
G \times_{P}\left(U_{P} \backslash A_{y_{r}}(P, P) / U_{P}\right) \stackrel{\sim}{\longrightarrow} Z_{J_{r}, y_{r}, \delta}^{\mathbf{s}_{r}}, \quad(g, \gamma) \mapsto\left({ }^{g} P,{ }^{g} P, g \gamma g^{-1}\right) .
$$

Now let $\vartheta: Z_{J, y, \delta}^{\mathbf{s}} \rightarrow Z_{J_{r}, y_{r}, \delta}^{\mathbf{s}_{r}}$ be a composition of maps in 8.20(a), a smooth map with connected fibres. Then $\tilde{X}=\tilde{\vartheta}\left(X^{\prime}\right)$ is a simple perverse sheaf on $Z_{J, y, \delta}^{\text {s }}$. Let $\hat{X}$ be the simple perverse sheaf on $Z_{J, y, \delta}$ whose support is the closure in $Z_{J, y, \delta}$ of $\operatorname{supp} \tilde{X}$ and whose restriction to $Z_{J, y, \delta}^{\mathrm{s}}$ is $\tilde{X}$.

Let $\mathcal{C}_{J, y, \delta}^{\prime} \mathbf{s}$ be the class of simple perverse sheaves on $Z_{J, y, \delta}^{\mathbf{s}}$ consisting of all $\tilde{X}$ as above. Let $\mathcal{C}_{J, y, \delta}^{\prime}$ be the class of simple perverse sheaves on $Z_{J, y, \delta}$ consisting of all $\hat{X}$ as above (where s varies). The isomorphism classes of objects in $\mathcal{C}_{J, y, \delta}^{\prime}$ are in bijection with the set of pairs $(\mathbf{s}, X)$ where $\mathbf{s} \in S(J, \operatorname{Ad}(y) \delta)$ and $X$ is a character sheaf on $C^{\mathbf{s}}$ (as above).

Lemma 11.13. Let $\mathbf{s}=\left(J_{n}, J_{n}^{\prime}, u_{n}\right)_{n \geq 0} \in S(J, \operatorname{Ad}(y) \delta)$. Let $\mathcal{L} \in \mathcal{S}(T)$ and let $w \in W$ be such that $w y \in W_{\mathcal{L}}^{1}$; let $\tilde{\mathcal{L}}$ be the corresponding local system on ${ }^{y} Y_{w, y}^{\prime}$. Let $A^{\prime}$ be a simple perverse sheaf on $Z_{J, y, \delta}^{\mathbf{s}}$ such that $\left.A^{\prime} \dashv\left(\Pi_{w, y}\right) ! \tilde{\mathcal{L}}\right|_{Z_{J, y, \delta}^{\mathbf{s}}}$. Then $A^{\prime} \in \mathcal{C}_{J, y, \delta}^{\prime}$.

More generally, we show that the lemma holds when $J, \mathbf{s}$ are replaced by $J_{n}, \mathbf{s}_{n}$ for any $n \geq 0$. First we show:

(a) if the result is true for $n=1$ then it is true for $n=0$.

Let $\mathcal{L}^{\prime}, \tilde{\mathcal{L}}^{\prime}$ be related to $\mathcal{L}, \tilde{\mathcal{L}}$ as in 11.6. The restrictions of $\tilde{\mathcal{L}}, \tilde{\mathcal{L}}^{\prime}$ to ${ }^{y} Y_{w, y}^{\prime}$, ${ }^{z^{-1} y} Y_{\delta^{-1}\left(b^{\prime}\right), a z, z^{-1} y}^{\mathbf{s}_{1}}\left(\right.$ where $\left.z=u_{0}\right)$ are again denoted by $\tilde{\mathcal{L}}, \tilde{\mathcal{L}}^{\prime}$. In the last cartesian diagram in $11.10, \tilde{\mathcal{L}}$ is the inverse image of $\tilde{\mathcal{L}}^{\prime}$ under

$$
{ }^{y} Y_{w, y}^{\prime}{ }^{\mathbf{s}} \rightarrow{ }^{z^{-1} y} Y_{\delta^{-1}\left(b^{\prime}\right), a z, z^{-1} y}^{\prime \mathbf{s}_{1}}
$$

(see the last sentence in 11.8). By the change of basis theorem, the direct image with compact support of $\tilde{\mathcal{L}}$ under ${ }^{y} Y_{w, y}^{\prime} \mathbf{s} \rightarrow Z_{J, y, \delta}^{\mathbf{s}}$ is $\vartheta^{*}(\vartheta$ as in 8.19$)$ of the direct image with compact support of $\tilde{\mathcal{L}}^{\prime}$ under

$$
{ }^{z^{-1} y} Y_{\delta^{-1}\left(b^{\prime}\right) a z, z^{-1} y}^{\mathbf{s}_{1}} \rightarrow Z_{J_{1}, z^{-1} y, \delta}^{\mathbf{s}_{1}}
$$

In other words,

$$
\left(\Pi_{w, y}\right),\left.\tilde{\mathcal{L}}\right|_{Z_{J, y, \delta}^{\mathrm{s}}}=\vartheta^{*}\left(\left.\left(\Pi_{\delta^{-1}\left(b^{\prime}\right), a z, z^{-1} y}\right) ! \tilde{\mathcal{L}}^{\prime}\right|_{Z_{J_{1}, z^{-1} y, \delta}^{\mathbf{s}_{1}}}\right) .
$$


Thus, $A^{\prime} \dashv \vartheta^{*}\left(\left.\left(\Pi_{\delta^{-1}\left(b^{\prime}\right), a z, z^{-1} y}\right) ! \tilde{\mathcal{L}}^{\prime}\right|_{Z_{J_{1}, z^{-1} y, \delta}^{\mathbf{s}_{1}}}\right)$. Since $\vartheta$ is an affine space bundle it follows that there exists a simple perverse sheaf $A^{\prime \prime}$ on $Z_{J_{1}, z^{-1} y, \delta}^{\mathbf{s}_{1}}$ such that $A^{\prime}=\tilde{\vartheta}\left(A^{\prime \prime}\right)$ and $\left.A^{\prime \prime} \dashv\left(\Pi_{\delta^{-1}\left(b^{\prime}\right), a z, z^{-1} y}\right) ! \tilde{\mathcal{L}}^{\prime}\right|_{Z_{J_{1}, z^{-1} y, \delta}^{s_{1}}}$. Applying 11.11(a) for $\delta^{-1}\left(b^{\prime}\right), a z, z^{-1} y, \mathbf{s}_{1}$ instead of $x_{1}, x_{2}, y$, $\mathbf{s}$ we see that there exists $x_{0} \in W$ such that $x_{0} z^{-1} y \in W_{\mathcal{L}^{\prime}}^{1}$ and $A^{\prime \prime} \dashv\left(\left.\left(\Pi_{x_{0}, y} \tilde{\mathcal{L}}^{\prime}\right)\right|_{Z_{J_{1}, z^{-1} y, \delta}^{\mathrm{s}_{1}}}\right.$. By our assumption we have $A^{\prime \prime} \in \mathcal{C}_{J_{1}, z^{-1} y, \delta}^{\prime \mathbf{s}_{1}}$. From the definitions we have $\tilde{\vartheta}\left(A^{\prime \prime}\right) \in \mathcal{C}_{J, y, \delta}^{\prime}$. Thus, (a) holds.

Similarly, if the result holds for some $n \geq 1$ then it holds for $n-1$. (The proof is the same as for $n=1$.) In this way we see that it suffices to prove the result for $n \gg 0$. Thus we may assume that $J_{0}=J_{0}^{\prime}=J_{1}=J_{1}^{\prime}=\cdots=J$ and $u_{0}=u_{1}=\cdots=1$. Then $W_{J} y=y W_{\delta(J)}$. In our case, ${ }^{y} Y_{w, y}^{\prime}{ }^{\mathbf{s}} \neq \emptyset$ hence there exist $B_{0}, B_{1} \in \mathcal{B}$ such that $\operatorname{pos}\left(B_{0}, B_{1}\right)=w$ and $B_{0}, B_{1}$ are contained in the same parabolic of type $J$. Thus we have $w \in W_{J}$. Let $P \in \mathcal{P}_{J}$ with $T \subset P$. Let $L$ be the Levi of $P$ such that $T \subset L$. Then

$$
C=\left\{c \in G^{1} ;{ }^{c} L=L, \operatorname{pos}\left(P,{ }^{c} P\right)=y\right\}
$$

is a connected component of $N_{\hat{G}}(L)$. Let $Y^{\prime}$ be the set of all $\left(\beta_{0}, \beta_{1}, c\right)$ where $\beta, \beta^{\prime}$ are Borels of $L$ such that $\operatorname{pos}\left(\beta_{0}, \beta_{1}\right)=w$ (position with respect to $L$ with Weyl group $W_{J}$ ) and $c \in C$ is such that ${ }^{c} \beta_{0}=\beta_{1}$. Then $P$ acts on $Y^{\prime}$ by $p:\left(\beta_{0}, \beta_{1}, c\right) \mapsto\left({ }^{l} \beta_{0},{ }^{l} \beta_{1},{ }^{l} c\right)$ where $l \in L, p \in l U_{P}$. We have a commutative diagram

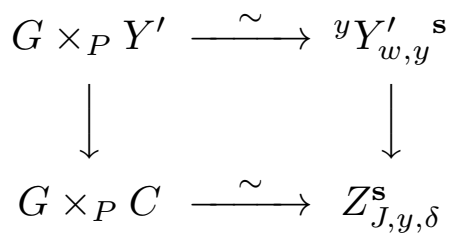

where the upper horizontal map is

$$
\left(g, \beta_{0}, \beta_{1}, c\right) \mapsto\left({ }^{g} B_{0},{ }^{g} B_{1},{ }^{g} c\right)
$$

with $B_{0}=\beta_{0} U_{P}, B_{1}=\beta_{1} U_{P}$, the lower horizontal map is

$$
(g, c) \mapsto\left({ }^{g} P,{ }^{g} P, U_{P} g c g^{-1} U_{P}\right),
$$

the left vertical map is $\left(g, \beta_{0}, \beta_{1}, c\right) \mapsto(g, c)$ and the right vertical map is $\Pi_{w, y}$. This commutative diagram shows that any composition factor of $\oplus_{i}^{p} H^{i}\left(\left(\Pi_{w, y}\right) ! \tilde{\mathcal{L}}\right)$ is of the form $X^{\prime}$ (notation of 11.12) where $X$ is a character sheaf on $C$; hence it is in $\mathcal{C}_{J, y, \delta}^{\prime} \mathbf{s}$. The lemma is proved.

Lemma 11.14. For $\mathbf{s} \in S(J, \operatorname{Ad}(y) \delta), A \in \mathcal{C}_{J, y, \delta}$, we set $A^{\mathbf{s}}=\left.A\right|_{Z_{J, y, \delta}^{\mathbf{s}}}$. Then any composition factor of $\oplus_{i}{ }^{p} H^{i}\left(A^{\mathbf{s}}\right)$ belongs to $\mathcal{C}_{J, y, \delta}^{\prime} \mathbf{s}$.

We can find $\mathcal{L} \in \mathcal{S}(T)$ and $\mathbf{x}=\left(x_{1}, x_{2}, \ldots, x_{r}\right)$ as in 11.1(b) such that $x_{1} x_{2} \ldots x_{r} \in$ $W_{\mathcal{L}}^{1}$ and $A \dashv \overline{\mathcal{K}}_{\mathbf{x}}^{\mathcal{L}}$ (see 11.1, 11.2). Since the complex $\overline{\mathcal{K}}_{\mathbf{x}}^{\mathcal{L}}$ is semisimple (see 11.1) 
we have $\overline{\mathcal{K}}_{\mathbf{x}}^{\mathcal{L}} \cong A[m] \oplus K^{\prime}$ for some $K^{\prime} \in \mathcal{D}\left(Z_{J, y, \delta}\right)$ and some $m \in \mathbf{z}$. Hence $\left.\overline{\mathcal{K}}_{\mathbf{x}}^{\mathcal{L}}\right|_{Z_{J, y, \delta}^{\mathrm{s}}} \cong A^{\mathbf{s}}[m] \oplus K_{1}^{\prime}$ for some $K_{1}^{\prime} \in \mathcal{D}\left(Z_{J, y, \delta}^{\mathrm{s}}\right)$. It suffices to show that, if $\left.A^{\prime} \dashv \mathcal{K}_{\mathbf{x}}^{\mathcal{L}}\right|_{Z_{J, y, \delta}^{\mathbf{s}}}$, then $A^{\prime} \in \mathcal{C}_{J, y, \delta}^{\prime}$. As in [L3, 2.11-2.16] we see that there exists $\mathcal{L} \in \mathcal{S}(T), w \in W$ such that $w y \in W_{\mathcal{L}}^{1}$ and $\left.A^{\prime} \dashv \mathcal{K}_{w, y}^{\mathcal{L}}\right|_{Z_{J, y, \delta}^{\mathrm{s}}}$. Using Lemma 11.13 we have $A^{\prime} \in \mathcal{C}_{J, y, \delta}^{\prime}$. The lemma is proved.

Lemma 11.15. If $A \in \mathcal{C}_{J, y, \delta}$ then $A \in \mathcal{C}_{J, y, \delta}^{\prime}$.

Since $Z_{J, y, \delta}=\sqcup_{J \subset I} Z_{J, y, \delta}^{\mathbf{s}}$, we can find $\mathbf{s} \in S(J, \operatorname{Ad}(y) \delta)$ such that $\operatorname{supp}(A) \cap$ $Z_{J, y, \delta}^{\mathbf{s}}$ is open dense in $\operatorname{supp}(A)$. Then $A^{\mathbf{s}}=\left.A\right|_{Z_{J, y, \delta}^{\mathbf{s}}}$ is a simple perverse sheaf on $Z_{J, y, \delta}^{\mathbf{s}}$ and $A^{\mathbf{s}} \in \mathcal{C}_{J, y, \delta}^{\prime} \mathbf{s}$ (Lemma 11.14). Now $A, A^{\mathbf{s}}$ are related just as $\hat{X}, \tilde{X}$ are related in 11.12. Hence $A \in \mathcal{C}_{J, y, \delta}^{\prime} \mathbf{s}$. The lemma is proved.

Lemma 11.16. Let $\mathbf{s}=\left(J_{n}, J_{n}^{\prime}, u_{n}\right)_{n \geq 0} \in S(J, \operatorname{Ad}(y) \delta), C, X, X^{\prime}, \tilde{X}, \hat{X}$ be as in 11.12. For any $n \geq 0$ define a simple perverse sheaf $X_{n}^{\prime}$ on $Z_{J_{n}, y_{n}, \delta}^{\mathbf{s}_{n}}$ by $X_{n}^{\prime}=$ $\tilde{\vartheta}\left(X_{n+1}^{\prime}\right)$ where $\vartheta: Z_{J_{n}, y_{n}, \delta}^{\mathbf{s}_{n}} \rightarrow Z_{J_{n+1}, y_{n+1}, \delta}^{\mathbf{s}_{n+1}}$ is as in 8.20(a) for $n \geq 0$ and $X_{n}^{\prime}=X^{\prime}$ for $n \gg 0$. Define $a_{n} \in W^{J_{n}^{\prime}}$ by $a_{n}^{-1}=u_{n} u_{n+1} \ldots u_{m}$ for $m \gg 0$. For any $n \geq 0$ there exists $\mathcal{L}_{n} \in \mathcal{S}(T)$ and $b_{n}^{\prime} \in W_{\delta\left(J_{\infty}\right)}$ (see 2.6) such that $a_{n} y_{n} b_{n}^{\prime} \in W_{\mathcal{L}_{n}}^{1}$ and $\left.X_{n}^{\prime} \dashv\left(\Pi_{a_{n} y_{n} b_{n}^{\prime} y_{n}^{-1}, y_{n}}\right) ! \tilde{\mathcal{L}}_{n}\right|_{Z_{J_{n}, y_{n}, \delta}^{\mathbf{s}_{n}}}$.

Assume that the result holds for $n=1$; we show that it holds for $n=0$. By assumption we have $X_{1}^{\prime} \dashv\left(\left.\Pi_{\left.a y b_{1}^{\prime} y^{-1} z, z^{-1} y\right) ! \tilde{\mathcal{L}}^{\prime}}\right|_{Z_{J_{1}, y_{1}, \delta}^{s_{1}}}\right.$ where $\tilde{\mathcal{L}}^{\prime}=\tilde{\mathcal{L}}_{1}, z=u_{0}, a=$ $a_{0}$. (We have $a_{1} y_{1}=a y$.) We consider the cartesian diagram in 11.10 with $w=a y b^{\prime} y^{-1}$ where $\delta^{-1}\left(b^{\prime}\right) a=a y b_{1}^{\prime} y^{-1}$. (We have $b^{\prime}=\delta\left(a y b_{1}^{\prime} y^{-1} a^{-1}\right) \in W_{\delta\left(J_{\infty}\right)}$ by 2.6.) The inverse image of $\tilde{\mathcal{L}}^{\prime}$ under ${ }^{y} Y_{w, y}^{\prime}{ }^{\mathbf{s}} \rightarrow{ }^{z^{-1} y} Y_{\delta^{-1}\left(b^{\prime}\right) a z, z^{-1} y} \mathbf{s}_{1}$ is $\tilde{\mathcal{L}}$ for some $\mathcal{L} \in \mathcal{S}(T)$ (see 11.8). Using the change basis theorem for the cartesian diagram in 11.10 we deduce that $\left.X_{0}^{\prime} \dashv\left(\Pi_{a y b^{\prime} y^{-1}, y}\right) ! \tilde{\mathcal{L}}\right|_{Z_{J, y, \delta}^{\mathbf{s}}}$.

The same argument shows that, if the result holds for some $n \geq 1$ then it also holds for $n-1$. In this way it suffices to show that the result holds for $n \gg 0$. Replacing $\mathbf{s}, n$ by $\mathbf{s}_{n}, 0$, we may assume that $J_{0}=J_{0}^{\prime}=J_{1}=J_{1}^{\prime}=\cdots=J$, $u_{0}=u_{1}=\cdots=1$ and $n=0$. Let $P, L$ be as in 11.13. We can find $w \in W_{J}$ such that $X \dashv\left(p r_{3}\right)$ ! $\tilde{\mathcal{L}}$ where $p r_{3}: Y^{\prime} \rightarrow C$ is defined with $Y^{\prime}$ as in 11.13 in terms of $w$; here $\mathcal{L} \in \mathcal{S}(T)$ and $\tilde{\mathcal{L}}$ is the corresponding local system on $Y^{\prime}$. Using the commutative diagram in 11.13 we see that $X^{\prime} \dashv\left(\Pi_{y b^{\prime} y^{-1}, y}\right)$ ! $\left.\tilde{\mathcal{L}}\right|_{Z_{J, y, \delta}}$ where $b^{\prime}=y^{-1} w y \in W_{\delta(J)}$ and $\tilde{\mathcal{L}}$ is the local system on ${ }^{y} Y_{w, y}^{\prime}$ s corresponding to $\mathcal{L}$. The lemma is proved.

Lemma 11.17. Let $\mathbf{s}=\left(J_{n}, J_{n}^{\prime}, u_{n}\right)_{n \geq 0} \in S(J, \operatorname{Ad}(y) \delta)$. Define $a \in W^{J^{\prime}}$ by $a^{-1}=u_{0} u_{1} \ldots u_{m}$ for $m \gg 0$. Let $b^{\prime} \in W_{\delta\left(J_{\infty}\right)}$. Then the image of $\Pi_{a y b^{\prime} y^{-1}, y}$ : ${ }^{y} Y_{a y b^{\prime} y^{-1}, y}^{\prime} \rightarrow Z_{J, y, \delta}$ is contained in $Z_{J, y, \delta}^{\mathbf{s}}$.

Let $\left(B_{0}, B_{1}, \gamma\right) \in{ }^{y} Y_{a y b^{\prime} y^{-1}, y}^{\prime}$. Let $\left(P, P^{\prime}, \gamma\right)=\Pi_{a y b^{\prime} y^{-1}, y}\left(B_{0}, B_{1}, \gamma\right)$. We have 
$y b^{\prime-1} y^{-1} \in W_{J^{\prime}}$. Hence

$$
\begin{aligned}
& \operatorname{pos}\left(P^{\prime}, P\right)=\min \left(W_{J^{\prime}} \operatorname{pos}\left(B_{1}, B_{0}\right) W_{J}\right)=\min \left(W_{J^{\prime}} y b^{\prime-1} y^{-1} a^{-1} W_{J}\right) \\
& =\min \left(W_{J^{\prime}} a^{-1} W_{J}\right)=u_{0} .
\end{aligned}
$$

Thus, $\left(P, P^{\prime}, \gamma\right) \in Z_{J, y, \delta ; z}$ where $z=u_{0}$. Let $\left(P_{1}, P_{1}^{\prime}, \gamma_{1}\right)$ be the image of $\left(P, P^{\prime}, \gamma\right)$ under $Z_{J, y, \delta ; z} \rightarrow Z_{J_{1}, z^{-1} y, \delta}$ (see 11.5). By the cartesian diagram in 11.8, there exists

$$
\left(\tilde{B}_{0}, \tilde{B}_{1}, \tilde{\gamma}\right) \in{ }^{z^{-1} y} Y_{\delta^{-1}\left(b^{\prime}\right) a z, z^{-1} y}^{\prime}
$$

such that

$$
\Pi_{\delta^{-1}\left(b^{\prime}\right) a z, z^{-1} y}\left(\tilde{B}_{0}, \tilde{B}_{1}, \tilde{\gamma}\right)=\left(P_{1}, P_{1}^{\prime}, \gamma_{1}\right) .
$$

We have $\delta^{-1}\left(b^{\prime-1}\right) \in W_{J_{\infty}} \subset W_{J_{1}}$ hence

$$
\begin{aligned}
& \operatorname{pos}\left(P_{1}^{\prime}, P_{1}\right)=\min \left(W_{J_{1}^{\prime}} \operatorname{pos}\left(\tilde{B}_{1}, \tilde{B}_{0}\right) W_{J_{1}}\right)=\min \left(W_{J_{1}^{\prime}} z^{-1} a^{-1} \delta^{-1}\left(b^{\prime-1}\right) W_{J_{1}}\right)= \\
& \min \left(W_{J_{1}^{\prime}} u_{0}^{-1} a^{-1} W_{J_{1}}\right)=u_{1} .
\end{aligned}
$$

Now $\delta^{-1}\left(b^{\prime}\right) a z=(a z)\left(z^{-1} y\right) b_{1}^{\prime}\left(y^{-1} z\right)$ where $b_{1}^{\prime}=(a y)^{-1} \delta^{-1}\left(b^{\prime}\right) a y \in W_{\delta\left(J_{\infty}\right)}$ (see 2.6). Hence in the previous argument we may replace $B_{0}, B_{1}, \gamma, P, P^{\prime}, a, y, b^{\prime}$ by $\tilde{B}_{0}, \tilde{B}_{1}, \tilde{\gamma}, P_{1}, P_{1}^{\prime}, a u_{0}, u_{0}^{-1} y, b_{1}^{\prime}$ and we see that the image $\left(P_{2}, P_{2}^{\prime}, \gamma_{2}\right)$ of $\left(P_{1}, P_{1}^{\prime}, \gamma_{1}\right)$ under $Z_{J_{1}, u_{0}^{-1} y, \delta ; u_{1}} \rightarrow Z_{J_{2}, u_{1}^{-1} u_{0}^{-1} y, \delta}$ satisfies $\operatorname{pos}\left(P_{2}^{\prime}, P_{2}\right)=u_{2}$. Continuing this process we find that $\left(P, P^{\prime}, \gamma\right) \in Z_{J, y, \delta}^{\mathbf{s}}$. The lemma is proved.

Lemma 11.18. If $A \in \mathcal{C}_{J, y, \delta}^{\prime}$ then $A \in \mathcal{C}_{J, y, \delta}$.

Let $\mathbf{s}, \tilde{X}, \hat{X}$ be as in the proof of Lemma 11.16. We may assume that $A=\hat{X}$. By Lemma 11.16 we have $\left.\tilde{X} \dashv\left(\Pi_{a y b^{\prime} y^{-1}, y}\right) ! \tilde{\mathcal{L}}\right|_{Z_{J, y, \delta}^{\text {s }}}$ where $a=a_{0}$ (see 11.16), $b^{\prime} \in W_{\delta\left(J_{\infty}\right)}$ for some $\mathcal{L} \in \mathcal{S}(T)$ with $a y b^{\prime} \in W_{\mathcal{L}}^{1}$. By Lemma 11.17, $\Pi_{a y b^{\prime} y^{-1}, y}$ : ${ }^{y} Y_{a y b^{\prime} y^{-1}, y}^{\prime} \rightarrow Z_{J, y, \delta}$ factors through a map $\Pi^{\prime}:{ }^{y} Y_{a y b^{\prime} y^{-1}, y}^{\prime} \rightarrow Z_{J, y, \delta}^{\mathbf{s}}$ and $\tilde{X}=\Pi_{!}^{\prime} \tilde{\mathcal{L}}$. Thus there exists a simple perverse sheaf on $Z_{J, y, \delta}$ whose support is the closure in $Z_{J, y, \delta}$ of $\operatorname{supp}(\tilde{X})$, whose restriction to $Z_{J, y, \delta}^{\mathrm{s}}$ is $\tilde{X}$ and which is a composition factor of $\oplus_{i}^{p} H^{i}\left(\left(\Pi_{a y b^{\prime} y^{-1}, y}\right) ! \tilde{\mathcal{L}}\right)$; this is necessarily $\hat{X}$. We see that $\hat{X} \in \mathcal{C}_{J, y, \delta}$. The lemma is proved.

11.19. For $P \in \mathcal{P}_{J}$ let $H_{P}$ be the inverse image of the connected centre of $P / U_{P}$ under $P \rightarrow P / U_{P}$. For $P, \tilde{P} \in \mathcal{P}_{J}$, the groups $H_{P} / U_{P}, H_{\tilde{P}} / U_{\tilde{P}}$ are canonically isomorphic (an element $h \in G$ that conjugates $P$ into $\tilde{P}$ induces an isomorphism $H_{P} / U_{P} \stackrel{\sim}{\longrightarrow} H_{\tilde{P}} / U_{\tilde{P}}$ that is independent of the choice of $h$ ). Thus we may identify the groups $H_{P} / U_{P}$ (with $P \in \mathcal{P}_{J}$ ) with a single torus $\Delta_{J}$ independent of $P$. Now $\Delta_{J}$ acts (freely) on $Z_{J, y, \delta}$ by $\delta:\left(P, P^{\prime}, \gamma\right) \mapsto\left(P, P^{\prime}, \gamma z\right)$ where $z \in H_{P}$ represents $\delta \in \Delta_{J}$ and each piece $Z_{J, y, \delta}^{\mathbf{s}}$ is $\Delta_{J}$-stable. We set

$$
\bar{Z}_{J, y, \delta}^{\mathrm{s}}=\Delta_{J} \backslash Z_{J, y, \delta}^{\mathrm{s}}, \bar{Z}_{J, y, \delta}=\Delta_{J} \backslash Z_{J, y, \delta}=\sqcup_{\mathbf{s}} \bar{Z}_{J, y, \delta}^{\mathbf{s}}
$$


The action of $G$ on $Z_{J, y, \delta}$ and on $Z_{J, y, \delta}^{\mathrm{s}}$ commutes with the action of $\Delta_{J}$ and induces an action of $G$ on $\bar{Z}_{J, y, \delta}$ and on $\bar{Z}_{J, y, \delta}^{\mathrm{s}}$.

For $\left(P, P^{\prime}, \gamma\right) \in Z_{J, y, \delta}$ we have naturally

$$
H_{P} / U_{P} \subset H_{P_{1}} / U_{P_{1}} \subset \ldots \subset H_{P_{r}} / U_{P_{r}} \subset \ldots
$$

hence we may identify $\Delta_{J}$ with a (closed) subgroup of the centre of $L^{\mathbf{s}}$ (as above). The bijection 8.21 (a) induces a bijection

$$
G \backslash \bar{Z}_{J, y, \delta} \leftrightarrow \sqcup_{\mathbf{s} \in S(J, \operatorname{Ad}(y) \delta)}\left(L^{\mathbf{s}} / \Delta_{J}\right) \backslash\left(C^{\mathbf{s}} / \Delta_{J}\right) .
$$

Here $C^{\mathbf{s}} / \Delta_{J}$ is the orbit space for the free action of $\Delta_{J}$ by right translation on $C^{\mathbf{s}}$ (restriction of the action of the centre of $L^{\mathbf{s}}$ by right translation) and the action of $L^{\mathbf{s}} / \Delta_{J}$ on $C^{\mathbf{s}} / \Delta_{J}$ is induced by the conjugation action of $L^{\mathbf{s}}$ on $C^{\mathbf{s}}$.

\section{Completion}

12.1. Assume that $P, P^{\prime}$ are two parabolics of $G$ (as in 0.1) and that $\Phi: P / U_{P} \rightarrow$ $P^{\prime} / U_{P^{\prime}}$ is an isomorphism of algebraic groups. Let $H_{P, P^{\prime}}^{\Phi}$ be the set of all $\left(f, f^{\prime}\right) \in$ $P \times P^{\prime}$ such that $\Phi$ carries the image of $f$ in $P / U_{P}$ to the image of $f^{\prime}$ in $P^{\prime} / U_{P^{\prime}}$. Clearly, $H_{P, P^{\prime}}^{\Phi}$ is a closed connected subgroup of $G \times G$ of dimension $\operatorname{dim} U_{P}+$ $\operatorname{dim} U_{P^{\prime}}+\operatorname{dim}\left(P / U_{P}\right)=\operatorname{dim} G$ and $P, P^{\prime}, \Phi$ can be reconstructed from $H_{P, P^{\prime}}^{\Phi}$.

Let $\mathcal{V}_{G}$ be the (projective) variety whose points are the $\operatorname{dim}(G)$-dimensional Lie subalgebras of Lie $(G \times G)$. We have Lie $H_{P, P^{\prime}}^{\Phi} \in \mathcal{V}_{G}$.

12.2. Assume now that we are in the setup of 3.1. Let $J, J^{\prime}, y$ be as in 8.8. For $\left(P, P^{\prime}, \gamma\right) \in Z_{J, y, \delta}$ we set $\Phi_{\gamma}=b a^{-1} \operatorname{Ad}(g): P / U_{P} \stackrel{\sim}{\rightarrow} P^{\prime} / U_{P^{\prime}}$ where $g \in \gamma$ and $a, b$ are the obvious isomorphisms in

$$
P / U_{P} \stackrel{\operatorname{Ad}(g)}{\longrightarrow}{ }^{g} P / U_{g_{P}} \stackrel{a}{\leftarrow}\left({ }^{g} P \cap P^{\prime}\right) / U_{g} P \cap P^{\prime} \stackrel{b}{\rightarrow} P^{\prime} / U_{P^{\prime}} .
$$

Now $\Phi_{\gamma}$ is independent of the choice of $g$ and $\left(P, P^{\prime}, \gamma\right) \mapsto$ Lie $H_{P, P^{\prime}}^{\Phi_{\gamma}}$ is an imbedding

$$
\bar{Z}_{J, y, \delta} \subset \mathcal{V}_{G}
$$

(notation of 11.19(a)).

12.3. In the remainder of this section we assume that $G$ is adjoint. Recall that two parabolics $Q, Q^{\prime}$ of $G$ are said to be opposed if their intersection is a common Levi of $Q, Q^{\prime}$. (We then write $Q \bowtie Q^{\prime}$.) If $B \in \mathcal{B}$ and $Q$ is a parabolic in $G$, we write $B \ltimes Q$ if $B$ is opposed to some Borel of $Q$.

Let $J \subset I$. Define $J^{*} \subset I$ by $\left\{Q ; Q \bowtie P\right.$ for some $\left.P \in \mathcal{P}_{J}\right\}=\mathcal{P}_{J^{*}}$. Let $y_{J}$ be the longest element in $W^{\delta(J)}$. If $\left(P, P^{\prime}, g\right) \in \mathcal{P}_{J} \times \mathcal{P}_{\delta(J)^{*}} \times G^{1}$, then $P^{\prime} \bowtie^{g} P$ if and only if $g \in A_{y_{J}}\left(P, P^{\prime}\right)$ (see 3.8). Let

$$
\bar{A}_{y_{J}}\left(P, P^{\prime}\right)=H_{P^{\prime}} \backslash A_{y_{J}}\left(P, P^{\prime}\right) / U_{P}=U_{P^{\prime}} \backslash A_{y_{J}}\left(P, P^{\prime}\right) / H_{P},
$$




$$
\begin{aligned}
G_{J}^{1}=\left\{\left(P, P^{\prime}, \mu\right) ; P \in \mathcal{P}_{J}, P^{\prime}\right. & \left.\in \mathcal{P}_{\delta(J)^{*}}, \mu \in \bar{A}_{y_{J}}\left(P, P^{\prime}\right)\right\}=\bar{Z}_{J, y_{J}, \delta}, \\
\bar{G}^{1} & =\sqcup_{J \subset I} G_{J}^{1} .
\end{aligned}
$$

(notation of $11.19(\mathrm{a})$ ). We define a structure of algebraic variety on $\bar{G}^{1}$. We identify $G_{J}^{1}$ with a subvariety of $\mathcal{V}_{G}$ by $12.2(\mathrm{a})$. Since $G$ is adjoint we have $G_{I}^{1}=G^{1}$. By $[\mathrm{DP}], \bar{G}^{1}$ is the closure of $G_{I}^{1}=G^{1}$ in $\mathcal{V}_{G}$, so that $\bar{G}^{1}$ is a projective variety. (In [DP] it is assumed that $G=G^{1}$ but the general case can be easily reduced to this special case; in fact, $\bar{G}^{1}$ is isomorphic to the analogous variety in the case $G=G^{1}$.) We have a partition

$$
\bar{G}^{1}=\sqcup_{J \subset I} \sqcup_{\mathbf{s} \in S\left(J, \operatorname{Ad}\left(y_{J}\right) \delta\right)} \bar{Z}_{J, y_{J}, \delta}^{\mathbf{s}}
$$

(see 11.19(a)) refining the partition $\bar{G}^{1}=\sqcup_{J \subset I} \Gamma_{J}^{1}$. Putting together the bijections $11.19(\mathrm{~b})$ we obtain a canonical bijection

$$
G \backslash \bar{G}^{1} \leftrightarrow \sqcup_{J \subset I} \sqcup_{\mathbf{s} \in S\left(J, \operatorname{Ad}\left(y_{J}\right) \delta\right)}\left(L^{\mathbf{s}} / \Delta_{J}\right) \backslash\left(C^{\mathbf{s}} / \Delta_{J}\right)
$$

Here the action of $G$ on $\bar{G}^{1}$ is the extension of the conjugation action of $G$ on $G^{1}$ and $\left(L^{\mathbf{s}} / \Delta_{J}\right) \backslash\left(C^{\mathbf{s}} / \Delta_{J}\right)$ is as in 11.19 .

Let $J \subset I, \mathbf{s} \in S\left(J, \operatorname{Ad}\left(y_{J}\right) \delta\right)$ and let $X$ be a character sheaf on $C^{\mathbf{s}}$ that is equivariant for the free action of $\Delta_{J}$ by right translation. The simple perverse sheaf $\tilde{X}$ on $Z_{J, y_{J}, \delta}^{\mathrm{s}}$ (see 11.12) is $\Delta_{J}$-equivariant hence it is a shift of the inverse image of a well defined simple perverse sheaf $\underline{\tilde{X}}$ on $\bar{Z}_{J, y_{J}, \delta}^{\mathrm{s}}$ under the canonical $\operatorname{map} Z_{J, y_{J}, \delta}^{\mathbf{s}} \rightarrow \bar{Z}_{J, y_{J}, \delta}^{\mathbf{s}}$. Let $\underline{\hat{X}}$ be the simple perverse sheaf on $\bar{G}^{1}$ whose support is the closure in $\bar{G}^{1}$ of $\operatorname{supp} \underline{\tilde{X}}$ and whose restriction to $\bar{Z}_{J, y_{J}, \delta}^{\mathrm{s}}$ is $\underline{\tilde{X}}$.

The character sheaves on $\bar{G}^{1}$ are by definition the simple perverse sheaves on $\bar{G}^{1}$ of the form $\underline{\hat{X}}$ with $X$ as above. The character sheaves on $\bar{G}^{1}$ are in bijection with the set of triples $(J, \mathbf{s}, X)$ where $J \subset I, \mathbf{s} \in S\left(J, \operatorname{Ad}\left(y_{J}\right) \delta\right)$ and $X$ is a character sheaf on $C^{\mathbf{s}}$ that is equivariant for the free action of $\Delta_{J}$ by right translation.

12.4. We now give another definition of a structure of algebraic variety on the set $\bar{G}^{1}$ which does not use $\mathcal{V}_{G}$. For $B, \tilde{B} \in \mathcal{B}$ and $J \subset I$, let

$$
G_{J}^{1, B, \tilde{B}}=\left\{\left(P, P^{\prime}, \mu\right) \in G_{J}^{1} ; B \ltimes P^{\prime}, \tilde{B} \ltimes P,{ }^{g}\left(P^{\tilde{B}}\right) \bowtie P^{\prime B}\right\}
$$

where $g \in \mu$ (an open subset of $\left.G_{J}^{1}\right)$. By the the substitution $\left(P, P^{\prime}, \mu\right) \mapsto$ $\left(B_{1}, B_{1}^{\prime}, \mu\right)$ where $B_{1}=P^{\tilde{B}}, B_{1}^{\prime}=P^{\prime B}$, we may identify $G_{J}^{1, B, \tilde{B}}$ with the set of all triples $\left(B_{1}, B_{1}^{\prime}, \mu\right)$ where $B_{1}, B_{1}^{\prime} \in \mathcal{B}, \operatorname{pos}\left(B, B_{1}^{\prime}\right)=y_{J^{*}}, \operatorname{pos}\left(\tilde{B}, B_{1}\right)=y_{\delta^{-1}(J)}$, $\mu \in \bar{A}_{y_{J}}\left(P, P^{\prime}\right)$ (with $P \in \mathcal{P}_{J}, P^{\prime} \in \mathcal{P}_{\delta(J)^{*}}$ defined by $B_{1} \subset P, B_{1}^{\prime} \subset P^{\prime}$ ) and ${ }^{g} B_{1} \bowtie B_{1}^{\prime}$ for some/any $g \in \mu$.

In particular, $G_{I}^{1, B, \tilde{B}}=\left\{g \in G^{1} ;{ }^{g} \tilde{B} \bowtie B\right\}$. Moreover, $G_{\emptyset}^{1, B, \tilde{B}}=\left\{\left(B_{1}, B_{1}^{\prime}\right) \in\right.$ $\left.\mathcal{B} \times \mathcal{B} ; B_{1} \bowtie \tilde{B}, B_{1}^{\prime} \bowtie B\right\}$ (we omit the $\mu$-component since it is uniquely determined). 
Define $\rho_{J}: G_{I}^{1, B, \tilde{B}} \rightarrow G_{J}^{1, B, \tilde{B}}$ by

$$
\rho_{J}(g)=\left({ }^{-1} P_{J},{ }^{g} \tilde{P}_{J}, g H_{\tilde{P}_{J}} g^{-1} U_{P_{J}} g\right)
$$

where $P_{J} \in \mathcal{P}_{\delta(J)}, \tilde{P}_{J} \in \mathcal{P}_{J^{*}}$ are given by $B \subset P_{J}, \tilde{B} \subset \tilde{P}_{J}$. This is well defined:

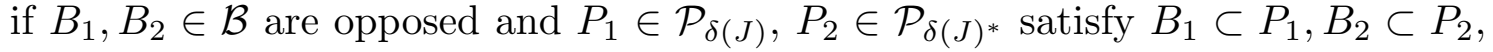
then $P_{1}^{B_{2}} \bowtie P_{2}^{B_{1}}$; apply this to $B_{1}=B, B_{2}={ }^{g} \tilde{B}$. that

In particular, $g \mapsto\left(g^{-1} B,{ }^{g} \tilde{B}\right)$ is a morphism $\rho_{\emptyset}: G_{I}^{1, B, \tilde{B}} \rightarrow G_{\emptyset}^{1, B, \tilde{B}}$. We show

(a) $\rho_{\emptyset}$ is a principal $\tilde{B} / U_{\tilde{B}}$-bundle.

We fix $\left(B_{1}, B_{1}^{\prime}\right) \in G_{\emptyset}^{1, B, \tilde{B}}$. Let $F=\rho_{\emptyset}^{-1}\left(B_{1}, B_{1}^{\prime}\right)$. Since $B_{1} \bowtie \tilde{B}$ and $B_{1}^{\prime} \bowtie B$, we can find $g_{0} \in G$ such that ${ }^{g_{0}} B_{1}=B,{ }^{g_{0}} \tilde{B}=B_{1}^{\prime}$. Next we can find $g_{1} \in G^{1}$ such that ${ }^{g_{1}} B=B,{ }^{g_{1}} B_{1}^{\prime}=B_{1}^{\prime}$. We have ${ }^{g^{-1} g_{0}} B_{1}=B,{ }^{g_{1}^{-1} g_{0}} \tilde{B}=B_{1}^{\prime}$. Hence $g_{1}^{-1} g_{0} \in F$ and $F \neq \emptyset$.

We show that $F$ is a free homogeneous $\tilde{B} / U_{\tilde{B}}$-space. Since $\tilde{B} \bowtie B_{1}$, we have canonically $\tilde{B} / U_{\tilde{B}}=\tilde{B} \cap B_{1}$. It suffices to show that $F$ is a free homogeneous $\tilde{B} \cap B_{1}$-space. Now $\tilde{B} \cap B_{1}$ acts freely on $F$ by $t: g \mapsto g t$. Let $g, g^{\prime} \in F$. Since ${ }^{g^{-1}} B={ }^{g^{\prime-1}} B$ and $g^{\prime} g^{-1} \in G$, we have $g^{\prime}=b g$ where $b \in B$. Since ${ }^{g} \tilde{B}={ }^{g} \tilde{B}$ and $g^{-1} g^{\prime} \in G$, we have $g^{\prime}=g \tilde{b}$ where $\tilde{b} \in \tilde{B}$. We have $\tilde{b}=g^{-1} g^{\prime}=g^{-1} b \in g^{-1} B=B_{1}$. Thus, $\tilde{b} \in \tilde{B} \cap B_{1}$. This proves (a).

Similarly,

(b) $\rho_{J}$ is a principal $H_{\tilde{P}_{J}} / U_{\tilde{P}_{J}}$-bundle. ( $\tilde{P}_{J}$ is as above.)

Using simple roots we identify $\tilde{B} / U_{\tilde{B}}=\left(\mathbf{k}^{*}\right)^{I}$ (we use again that $G$ is adjoint). Now $\left(\mathbf{k}^{*}\right)^{I}$ acts on $\mathbf{k}^{I}$ by multiplication on each factor. This may be regarded as an action of $\tilde{B} / U_{\tilde{B}}$ on $\mathbf{k}^{I}$. Using the principal $\tilde{B} / U_{\tilde{B}}$-bundle $\rho_{\emptyset}$ we may form the associated bundle

$$
X^{B, \tilde{B}}=G_{I}^{1, B, \tilde{B}} \times_{\tilde{B} / U_{\tilde{B}}} \mathbf{k}^{I}
$$

(a $\mathbf{k}^{I}$-bundle over the affine space $G_{\emptyset}^{1, B, \tilde{B}}$ ). Now $X^{B, \tilde{B}}$ is an affine space of dimension $\operatorname{dim}(G)$. We have an obvious partition $\mathbf{k}^{I}=\sqcup_{J \subset I}\left(\mathbf{k}^{*}\right)^{J}$ and each piece is $\tilde{B} / U_{\tilde{B}}$-stable. Hence there is a partition

$$
X^{B, \tilde{B}}=\sqcup_{J \subset I} X_{J}^{B, \tilde{B}}
$$

where $X_{J}^{B, \tilde{B}}=G_{I}^{1, B, \tilde{B}} \times_{\tilde{B} / U_{\tilde{B}}}\left(\mathbf{k}^{*}\right)^{J}$. We may identify $X_{J}^{B, \tilde{B}}$ with the orbit space of $G_{I}^{1, B, \tilde{B}}$ by $H_{\tilde{P}_{J}} / U_{\tilde{P}_{J}}$, hence (using (b)) with $G_{J}^{1, B, \tilde{B}}$. Thus, we may identify $X^{B, \tilde{B}}$ with the subset of $\sqcup_{J \subset I} G_{J}^{1}$ so that $X^{B, \tilde{B}} \cap G_{J}^{1}=G_{J}^{1, B, \tilde{B}}$. We show that for any $J$ we have

$$
G_{J}^{1}=\cup_{B, \tilde{B} \in \mathcal{B}} G_{J}^{1, B, \tilde{B}}
$$


Let $\left(P, P^{\prime}, \mu\right) \in G_{J}^{1}, g \in \mu$. Since ${ }^{g} P \bowtie P^{\prime}$, we can find Borels $B_{1} \subset P, B_{1}^{\prime} \subset P^{\prime}$ such that ${ }^{g} B_{1} \bowtie B_{1}^{\prime}$. We can find $B \in \mathcal{B}$ such that $B \ltimes P^{\prime}$ and $P^{\prime B}=B_{1}^{\prime}$. We can find $\tilde{B} \in \mathcal{B}$ such that $\tilde{B} \ltimes P$ and $P^{\tilde{B}}=B_{1}$. Then $\left(P, P^{\prime}, \gamma\right) \in G_{J}^{1, B, \tilde{B}}$. This proves (c).

From (c) we deduce

$$
\bar{G}^{1}=\cup_{B, \tilde{B} \in \mathcal{B}} X^{B, \tilde{B}}
$$

We can now define a structure of algebraic variety on $\bar{G}^{1}$ by declaring that $X^{B, \tilde{B}}$ is an open subvariety of $\bar{G}^{1}$ for any $B, \tilde{B} \in \mathcal{B}$. We see that $\bar{G}^{1}$ has a covering by open subsets isomorphic to the affine space of $\operatorname{dimension} \operatorname{dim}(G)$.

12.5. Let $V=\left\{\left(B, B^{\prime}, g\right) \in \mathcal{B} \times \mathcal{B} \times G^{1} ; B^{\prime} \bowtie^{g} B\right\}$. The fibre of $p r_{12}: V \rightarrow \mathcal{B} \times \mathcal{B}$ at $\left(B, B^{\prime}\right)$ is just $G_{I}^{1, B^{\prime}, B}$. There is a unique free action of the torus $\Delta_{\emptyset}$ (see 11.19) on $V$ whose restriction to $G_{I}^{1, B^{\prime}, B}$ is the action of $\Delta_{\emptyset}=B / U_{B}$ appearing in 12.4(a). By 12.4(a), this makes $V$ into a principal $\Delta_{\emptyset}$-bundle over

$$
\left\{\left(B, B^{\prime}, B_{1}, B_{1}^{\prime}\right) \in \mathcal{B}^{4} ; B_{1} \bowtie B, B_{1}^{\prime} \bowtie B^{\prime}\right\}
$$

As in 12.4, we form the associated bundle

$$
X=V \times_{\Delta_{\emptyset}} \mathbf{k}^{I}
$$

(a $\mathbf{k}^{I}$-bundle over (a)). For $\left(B, B^{\prime}\right) \in \mathcal{B} \times \mathcal{B}$, the inclusion $G_{I}^{1, B^{\prime}, B} \subset V$ gives rise to an inclusion $G^{1, B^{\prime}, B} \times_{\Delta_{\emptyset}} \mathbf{k}^{I} \subset V \times_{\Delta_{\emptyset}} \mathbf{k}^{I}$ that is, $X^{B^{\prime}, B} \subset X$. The subsets $X^{B^{\prime}, B}$ form a partition of $X$. Let $\pi: X \rightarrow \bar{G}^{1}$ be the morphism which induces for any $B, B^{\prime}$ the identity map $X^{B^{\prime}, B} \rightarrow X^{B^{\prime}, B}$. We have a partition

$$
X=\sqcup_{J \subset I} X_{J}
$$

where $X_{J}=V_{I} \times_{\Delta_{\emptyset}}\left(\mathbf{k}^{*}\right)^{J}$. We may identify $X_{J}$ with the orbit space of $X$ by $\Delta_{I-J}$ hence, using $12.4(\mathrm{~b})$, with the set of all quintuples $\left(B_{1}, B_{1}^{\prime}, B, B^{\prime}, \mu\right)$ where

$$
\left(B, B^{\prime}, B_{1}, B_{1}^{\prime}\right) \in \mathcal{B}^{4}, \operatorname{pos}\left(B^{\prime}, B_{1}^{\prime}\right)=y_{J^{*}}, \operatorname{pos}\left(B, B_{1}\right)=y_{\delta^{-1}(J)}, \mu \in \bar{A}_{y_{J}}\left(P, P^{\prime}\right)
$$

(with $P \in \mathcal{P}_{J}, P^{\prime} \in \mathcal{P}_{\delta(J) *}$ defined by $B_{1} \subset P, B_{1}^{\prime} \subset P^{\prime}$ ) and ${ }^{g} B_{1} \bowtie B_{1}^{\prime}$ for some/any $g \in \mu$. Now $\pi: X \rightarrow \bar{G}^{1}$ restricts to the map $X_{J} \rightarrow G_{J}^{1}$ given by $\left(B_{1}, B_{1}^{\prime}, B, B^{\prime}, \mu\right) \mapsto\left(P, P^{\prime}, \mu\right)$ (with $P, P^{\prime}$ as above).

For $w \in W$ we set $V^{w}=\left\{\left(B, B^{\prime}, g\right) \in V ; \operatorname{pos}\left(B, B^{\prime}\right)=w\right\}, X^{w}=V^{w} \times_{\Delta_{\emptyset}} \mathbf{k}^{I}$. The sets $X_{J}^{w}=X^{w} \cap X_{J}$ form a partition of $X$. If $\left(B_{1}, B_{1}^{\prime}, B, B^{\prime}, \mu\right) \in X_{J}^{w}$, then

$$
\operatorname{pos}\left(B_{1}, B\right)=y_{\delta^{-1}(J)}^{-1}, \operatorname{pos}\left(B, B^{\prime}\right)=w, \operatorname{pos}\left(B^{\prime}, B_{1}^{\prime}\right)=y_{J^{*}}, \operatorname{pos}\left(B_{1}^{\prime},{ }^{g} B_{1}\right)=y_{\emptyset}
$$


for $g \in \mu$. Hence $\left(B_{1}, B, B^{\prime}, B_{1}^{\prime},{ }^{g} B_{1}, g\right) \in Y_{\mathbf{x}}$ where $\mathbf{x}=\left(y_{\delta^{-1}(J)}^{-1}, w, y_{J^{*}}, y_{\emptyset}\right)$ (see $4.2)$.

Let $\mathcal{Y}_{\mathbf{x}}$ be the set of all $\left(\beta_{0}, \beta_{1}, \beta_{2}, \beta_{3}, \beta_{4}, \gamma\right)$ where $\left(\beta_{0}, \beta_{1}, \beta_{2}, \beta_{3}, \beta_{4}\right) \in \mathcal{B}^{5}$ and $\gamma \in U_{P^{\prime}} \backslash A_{y_{J}}\left(P, P^{\prime}\right) / U_{P}\left(\right.$ with $P \in \mathcal{P}_{J}, P^{\prime} \in \mathcal{P}_{\delta(J)^{*}}$ defined by $\left.\beta_{0} \subset P, \beta_{3} \subset P^{\prime}\right)$ are such that $\left(\beta_{0}, \beta_{1}, \beta_{2}, \beta_{3}, \beta_{4}, g\right) \in Y_{\mathbf{x}}$ for some/any $g \in \gamma$.

The obvious map $Y_{\mathbf{x}} \rightarrow \mathcal{Y}_{\mathbf{x}}$ is an affine space bundle. The obvious map $\mathcal{Y}_{\mathbf{x}} \rightarrow$ $X_{J}^{w}$ is a principal $\Delta_{\delta(J)}$-bundle. Let $\mathcal{L} \in \mathcal{S}(T)$ be such that $y_{\delta^{-1}(J)}^{-1} w y_{J^{*}} y_{\emptyset} \in W_{\mathcal{L}}^{1}$ and such that the associated local system $\tilde{\mathcal{L}}$ on $Y_{\mathbf{x}}$ is the inverse image under the composition $Y_{\mathbf{x}} \rightarrow \mathcal{Y}_{\mathbf{x}} \rightarrow X_{J}^{w}$ of a local system $\tilde{\mathcal{L}}_{0}$ on $X_{J}^{w}$. (In any case, $\tilde{\mathcal{L}}$ is the inverse image under $Y_{\mathbf{x}} \rightarrow \mathcal{Y}_{\mathbf{x}}$ of a well defined local system on $\mathcal{Y}_{\mathbf{x}}$ and we require that this last local system is $\Delta_{\delta(J)}$-equivariant.) Let $\mathbf{K}_{w, J}^{\mathcal{L}}$ be the direct image with compact support of $\tilde{\mathcal{L}}_{0}$ under $X_{J}^{w} \rightarrow \bar{G}^{1}$ (restriction of $\pi: X \rightarrow \bar{G}^{1}$ ).

We give a second definition of character sheaves on $\bar{G}^{1}$ as the simple perverse sheaves on $\bar{G}^{1}$ which are composition factors of $\oplus_{i}{ }^{p} H^{i}\left(\mathbf{K}_{w, J}^{\mathcal{L}}\right)$ for some $w, J, \mathcal{L}$ as above. We expect that this coincides with the definition in 12.3 .

12.6. There is a unique simple perverse sheaf $\mathbf{S}$ on $G^{1}$ such that:

(a) $\mathbf{S}$ is a direct summand of the perverse sheaf $\left(p r_{1}\right)_{!} \overline{\mathbf{Q}}_{l}[\operatorname{dim} G]$ where $p r_{1}$ : $\left\{(g, B) \in G^{1} \times \mathcal{B} ;{ }^{g} B=B\right\} \rightarrow G^{1}$ is the first projection (a small map);

(b) if $J \varsubsetneqq I, \delta(J)=J$, then $\mathbf{S}$ is not a direct summand of the perverse sheaf $\left(p r_{1}\right)$ ! $\overline{\mathbf{Q}}_{l}[\operatorname{dim} G]$ where $p r_{1}:\left\{(g, P) \in G^{1} \times \mathcal{P}_{J} ;{ }^{g} P=P\right\} \rightarrow G^{1}$ is the first projection (a small map).

Let $\tilde{\mathbf{S}}$ be the simple perverse sheaf on $\bar{G}^{1}$ such that $\left.\tilde{\mathbf{S}}\right|_{G^{1}}=\mathbf{S}$. For $x \in \bar{G}^{1}$ let $G_{x}$ be the stabilizer of $x$ in $G$ and let $\mathcal{H}_{x}^{i}(\tilde{\mathbf{S}})$ be the stalk at $x$ of the $i$-th cohomology sheaf of $\tilde{\mathbf{S}}$.

We conjecture that the following three conditions on $x \in \bar{G}^{1}$ are equivalent:

(c) $\mathcal{H}_{x}^{i}(\tilde{\mathbf{S}}) \neq 0$ for some $i$;

(d) $\sum_{i} \operatorname{dim} \mathcal{H}_{x}^{i}(\tilde{\mathbf{S}})=1$;

(e) $G_{x}$ is a reductive group.

If we assume that $x \in G^{1}$, then the equivalence of (c),(d),(e) is known.

12.7. A difficulty in proving the conjecture in 12.6 is that the small map in 12.6 (a) does not seem to extend to a small map over all of $\bar{G}^{1}$. There is one case when such an extension exists (partially). Assume that $G=G^{1}=P G L(V)$ where $V$ is a $\mathbf{k}$-vector space of dimension $d \geq 2$. Let

$$
Y=\{\tau \in \operatorname{End}(V) ; \operatorname{dim} \operatorname{ker}(\tau) \leq 1\} / \mathbf{k}^{*}
$$

where $\mathbf{k}^{*}$ acts by scalar multiplication. For $\tau$ as above let $\bar{\tau}$ be the image of $\tau$ in $Y$. Let $Y_{0}=\{\tau \in \operatorname{End}(V) ; \operatorname{dim} \operatorname{ker}(\tau)=1\} / \mathbf{k}^{*}$. We may identify $Y$ with an open subset of $\bar{G}^{1}$ so that $Y-Y_{0}$ corresponds to the open stratum $G^{1}$ and $Y_{0}$ corresponds to a codimension 1 stratum $G_{J_{0}}^{1}$. Let $\tilde{Y}$ be the set of all $\left(\bar{\tau}, V_{1} \subset V_{2} \subset \ldots \subset V_{d}\right)$ where $\bar{\tau} \in Y$ and $V_{1} \subset V_{2} \subset \ldots \subset V_{d}$ are subspaces of $V\left(\operatorname{dim} V_{i}=i\right)$ such that $\tau\left(V_{i}\right) \subset V_{i}$ for all $i$. Then $\tilde{Y}$ is smooth and $p r_{1}: \tilde{Y} \rightarrow Y$ is a small map. 
Its restriction to $\mathrm{pr}_{1}^{-1}(G)$ may be identified with the small map in 12.6(a). Let $K_{\emptyset}=\left(p r_{1}\right)_{!} \overline{\mathbf{Q}}_{l}[\operatorname{dim} G]$, a perverse sheaf on $Y$. More generally, for any $J \subset I$ we have a perverse sheaf $K_{J}$ on $Y$ defined like $K_{\emptyset}$ but using flags of type $J$ (see 8.24) in $V$ instead of complete flags. Let $\tilde{\mathbf{S}}^{\prime}=\left.\tilde{\mathbf{S}}\right|_{Y}$ that is, the simple perverse sheaf on $Y$ such that $\left.\tilde{\mathbf{S}}^{\prime}\right|_{G}=\mathbf{S}$. Now $\mathbf{S}$ is an alternating sum over $J$ of the perverse sheaves $\left.K_{J}\right|_{G}$; hence $\tilde{\mathbf{S}}^{\prime}$ is an alternating sum over $J$ of the perverse sheaves $K_{J}$. Using this, one can compute explicitly the stalks of the cohomology sheaves of $\tilde{\mathbf{S}}^{\prime}$ at any $x \in Y_{0}$. Thus one can verify that the conjecture in 12.6 holds in our case for $x \in Y_{0}$.

\section{REFERENCES}

[B] R.Bédard, On the Brauer liftings for modular representations, J.Algebra 93 (1985), 332353.

[BBD] A.A.Beilinson, J.Bernstein and P.Deligne, Faisceaux pervers, Astérisque 100 (1982).

[DP] C.De Concini and C.Procesi, Complete symmetric varieties, Invariant theory (Montecatini 1982), Lect.Notes Math., vol. 996, Springer, 1983, pp. 1-44.

[L3] G.Lusztig, Character sheaves, I, Adv.Math. 56 (1985), 193-237.

[L9] G.Lusztig, Parabolic character sheaves, I.

Department of Mathematics, M.I.T., Cambridge, MA 02139 